\title{
Bond-order and entropic probes of the chemical bonds
}

\author{
Roman F. Nalewajski · Piotr Gurdek
}

Received: 28 February 2011 / Accepted: 26 October 2011/Published online: 7 June 2012

(C) The Author(s) 2012. This article is published with open access at Springerlink.com

\begin{abstract}
An overview of the recent bond-order and entropy/information measures of the chemical bond multiplicity and of its covalent/ionic composition is given. The former include the Wiberg index of the molecular orbital (MO) theory and its atomic/diatomic components, while the latter explore the communication-noise (covalency) and information-flow (ionic) descriptors of molecular information channels in the atomic-orbital (AO) resolution. The illustrative application to the two-orbital model is presented and the atomic resolution of bond contributions is presented. Alternative information distributions, including densities of the displacement in the system Shannon entropy and its entropy deficiency relative to the "promolecule," are advocated as effective probes of chemical bonds. They complement the familiar density difference diagrams of electron redistributions accompanying the bond formation process. These quantities are applied to investigate the central bond in small propellanes and the contragradience criterion, based upon the non-additive Fisher information in electron distribution, is shown to efficiently locate the bonding regions in butadiene and benzene. The novel, indirect bonding mechanism through the orbital intermediaries, inferred from the orbital communication theory in the AO resolution, is probed in these two illustrative $\pi$-electron systems using the generalized Wiberg bond-orders. It is shown to give rise to a more
\end{abstract}

This paper is devoted to Professor Malgorzata Witko.

Throughout the paper, $A$ denotes scalar quantity, $\boldsymbol{A}$ stands for the row or column vector, and $\mathbf{A}$ represents a rectangular or square matrix.

R. F. Nalewajski $(\bowtie) \cdot$ P. Gurdek

Department of Theoretical Chemistry, Jagiellonian University,

R. Ingardena 3, 30-060 Cracow, Poland

e-mail: nalewajs@chemia.uj.edu.pl realistic representation of the second-neighbor interactions, which have previously been diagnosed as the direct (through-space) non-bonding. In MO theory, these through-bridge bond components are due to the implicit dependencies between the (non-orthogonal) AO projections onto the molecular bonding subspace of the occupied MO. They do not require the bond-charge accumulation between the nuclei of bonded atoms and can be realized at longer distances. The effective range of such indirect interactions is probed in representative polymers. Finally, the entropy/information concepts for three dependent probability distributions are used to qualitatively examine the promotion of reactants in catalysis. The chemisorbed species are predicted to undergo an ionic promotion, compared to the gas-phase reference, thus exhibiting more deterministic communications on the catalytic surface.

Keywords Bond orders - Chemical bond multiplicities Contragradience criterion - Entropy/information descriptors - Direct (through space) bonding - Indirect (through bridge) bonds - Information theory - Orbital communications in molecules · Polymers - Wiberg bond multiplicity

\section{Introduction}

The electron redistribution accompanying the bond formation, from the electron density $\rho^{0}=\sum_{\mathrm{X}} \rho_{\mathrm{X}}^{0}$ due to molecularly placed free atoms $\left\{\mathrm{X}^{0}\right\}$ of the system "promolecule," exhibiting the ground-state densities $\left\{\rho_{\mathrm{X}}^{0}\right\}$, to the molecular distribution $\rho=\sum_{\mathrm{X}} \rho_{\mathrm{X}}$ generated by electron densities $\left\{\rho_{\mathrm{X}}\right\}$ of the bonded atoms $\{\mathrm{X}\}$, is marked by the familiar difference function, $\Delta \rho=\rho-\rho^{0}$ between these resultant molecular and promolecular electron densities. The 
entropy/information content of these distributions provides a basis for a novel information-theoretic (IT) perspective on the molecular electronic structure, e.g. [1-3] and references therein. For example, the densities of displacements in the Shannon entropy and of the missing information (entropy deficiency, cross-entropy) relative to the promolecular reference, have been used as sensitive diagnostic tools for detecting the chemical bonds and to monitor the promotion/ hybridization changes that the bonded atoms undergo in the molecular environment [4-6], while the alternative variational principles of IT [7-15] have been used [1] to extract unbiased "stockholder" atoms-in-molecules (AIM) of Hirshfeld [16].

The equilibrium distributions of electrons in molecular or reactive systems carry the associated information content, which changes during the chemical bond formation or in the course of a chemical reaction. The bonded atoms and larger molecular fragments (open subsystems) constantly exchange electrons, and hence also the information. In a sense, they "talk" to each other. It is thus a challenging task to describe and understand the information content of electronic probability distributions in molecules, reactive systems, and their subsystems and its changes in chemical processes. Such an IT approach provides an alternative perspective on the molecular electronic structure, similarity, and reactivity. In fact, an insight into the entropic origins of chemical bonds and their couplings in diverse chemical phenomena is central to many branches of chemistry, particularly to the theory of chemical reactivity. The quantum mechanical state of a molecule is determined by the system electronic wave-function, the (complex) amplitude of the associated probability distribution, which carries the information. It is intriguing to explore the information content of the electronic probability distribution in a molecule or in its amplitude, and to extract from it the pattern of chemical bonds, trends in chemical reactivity, and other molecular descriptors, e.g., the bond multiplicities ("orders") and their covalent and ionic components [1-3].

The spatial localization of specific bonds, not to mention some qualitative questions about the very existence of some controversial chemical bonds, e.g., between the bridgehead carbon atoms in small propellanes [1-3, 17, 18], presents another challenging problem for this novel IT treatment of molecular systems. The non-additive Fisher information in the $\mathrm{AO}$ resolution has been recently used as the contragradience (CG) criterion [2, 3, 15, 19-21], related to the kinetic energy of electrons, for localizing the bonding regions in molecules, while the related information density in the molecular orbital (MO) resolution has been shown [22] to determine the vital ingredient of the electron localization function (ELF) [23-25].

The orbital communication theory (OCT) of the chemical bond [2, 3, 26-34] uses the standard entropy/information descriptors of the Shannon theory of communications [7-10] to characterize the scattering of the AO electron probabilities, throughout the network of chemical bonds generated by the system occupied MO [26]. The molecule is thus treated as an information system [1-3, 35] which propagates, from the channel AO "inputs" to AO "outputs", the "signals" of the electron allocations to these basis functions of the molecular SCF LCAO MO calculations. The underlying conditional probabilities determining the communication network in molecules are generated from the (bond-projected) superposition principle of quantum mechanics $[26,36]$. They have been shown to be proportional to the squares of the corresponding elements of the first-order density matrix in the AO representation, thus being also related to the Wiberg [37] quadratic index of the chemical bond multiplicity and its subsequent generalizations [38-47].

Such information propagation in molecules exhibits a typical communication "noise" due to the electron delocalization via the system chemical bonds, which lowers the amount of information passing through the channel. The orbital information systems can used to generate the entropic measures of the chemical bond multiplicity and their covalent/ionic composition for both the molecule as a whole and its diatomic fragments. They also determine the entropy/information descriptors of an effective chemical coupling between such subsystems, individual chemical bonds, reactivities of different sites in reactive systems, etc. The average conditional entropy, which measures the channel communication noise due to electron delocalization (communication-indeterminacy), measures the ITcovalency in the molecule, while the complementary descriptor of the network mutual information (informationflow, communication-determinacy) reflects the electron localization effects and measures the system IT-ionic component [32, 35].

To summarize, the entropic probes of the molecular electronic structure have provided novel, attractive tools for describing the chemical bond phenomena in information terms. It is the main purpose of the next section to introduce the key concepts and techniques of IT which will be used in subsequent sections to illustrate some of the recent developments in alternative entropy/information probes of the molecular electronic structure and in OCT, the orbital formulation of the communication theory of the chemical bond (CTCB) [1]. The recently discovered mechanism of indirect chemical bonding [3, 18, 48-52] will be also emphasized.

\section{Rudiments of IT approach}

The Shannon [7,8] entropy content $S[p]$ in the (normalized) spatial probability distribution $p(\boldsymbol{r})$, 
$S[p] \equiv-\int p(\boldsymbol{r}) \log p(\boldsymbol{r}) \mathrm{d} \boldsymbol{r}, \quad \int p(\boldsymbol{r}) \mathrm{d} \boldsymbol{r}=1$,

where the definite integration is over the whole range of the electron position coordinates $\boldsymbol{r}$, provides a measure of the average indeterminacy (spread) in $p(\boldsymbol{r})$ for the locality events $\{\boldsymbol{r}\}$. It also measures the average amount of information obtained when this spatial uncertainty is removed by an appropriate localization measurement (experiment). Here the logarithm is taken to an arbitrary but fixed base: when taken to base $2, \log =\log _{2}$, the information is measured in bits, while selecting $\log =\ln$ expresses the information in nats: 1 nat $=1.44$ bits.

The Fisher [13] information $I[p]$ historically predates the Shannon entropy by about 25 years, being proposed in about the same time when the final form of the quantum mechanics was shaped. The intrinsic-accuracy functional for the locality parameter, reminiscent of von Weizsäcker's [53] inhomogeneity correction to the electronic kinetic energy in Thomas-Fermi theory,

$I[p]=\int p(\boldsymbol{r})[\nabla \ln p(\boldsymbol{r})]^{2} \mathrm{~d} \boldsymbol{r}=\int[\nabla p(\boldsymbol{r})]^{2} / p(\boldsymbol{r}) \mathrm{d} \boldsymbol{r}$,

characterizes the localization (compactness) of the probability density $p(\boldsymbol{r})$. For example, the Fisher information in the familiar normal distribution measures the inverse of its variance, called the invariance, while the complementary Shannon entropy is proportional to the logarithm of variance, thus monotonically increasing with the spread of the Gaussian distribution.

The form of Fisher functional can be simplified by expressing it in terms of the associated probability amplitude $\alpha(\boldsymbol{r})=\sqrt{p(\boldsymbol{r})}$ :

$I[p]=4 \int[\nabla \alpha(\boldsymbol{r})]^{2} \mathrm{~d} \boldsymbol{r} \equiv I[\alpha]$.

It is naturally generalized into a case of the complex probability amplitudes encountered in quantum mechanics [15], the system wave-functions. For the simplest case of the spinless one-particle system described by the wavefunction $\psi(\boldsymbol{r})$, when $p(\boldsymbol{r})=\psi^{*}(\boldsymbol{r}) \psi(\boldsymbol{r})=|\psi(\boldsymbol{r})|^{2}$,

$$
\begin{aligned}
I[\psi] & =4 \int|\nabla \psi(\boldsymbol{r})|^{2} \mathrm{~d} \boldsymbol{r}=4 \int \nabla \psi^{*}(\boldsymbol{r}) \cdot \nabla \psi(\boldsymbol{r}) \mathrm{d} \boldsymbol{r} \\
& \equiv \int f(\boldsymbol{r}) \mathrm{d} \boldsymbol{r} .
\end{aligned}
$$

Thus, $I[\alpha]$ or $I[\psi]$ measure the gradient content in the amplitude of the probability density and hence it is related to the kinetic energy of electrons [15].

An important generalization of Shannon's entropy, called the relative (cross) entropy, also known as the entropy deficiency, missing information or the directed divergence, has been proposed by Kullback and Leibler $[11,12]$. It measures the information "distance" between the two (normalized) probability distributions for the same set of events. For example, in the continuous probability scheme $\mathrm{A}=[\boldsymbol{r}, p(\boldsymbol{r})]$, involving the electron locality events $\{\boldsymbol{r}\}$ and their probabilities $\{p(\boldsymbol{r})\}$, this discrimination information in $p$ with respect to the reference distribution $p^{0}$ reads:

$\Delta S\left[p \mid p^{0}\right]=\int p(\boldsymbol{r}) \log \left[p(\boldsymbol{r}) / p^{0}(\boldsymbol{r})\right] \mathrm{d} \boldsymbol{r} \equiv \int \Delta s(\boldsymbol{r}) \mathrm{d} \boldsymbol{r} \geq 0$.

This referenced Shannon's entropy provides a measure of an information resemblance between the two compared probability schemes. The more the two probability distributions differ from one another, the larger the information distance [1].

Consider next the IT descriptors reflecting the dependency between probability distributions. For two dependent (discrete) probability schemes $\mathrm{A}=[\boldsymbol{a}, P(\boldsymbol{a})]$ and $\mathrm{B}=[\boldsymbol{b}, \boldsymbol{P}(\boldsymbol{b})]$ (see upper part of Fig. 1), defining the distributions $\boldsymbol{P}(\boldsymbol{a})=\left\{P\left(a_{i}\right)=p_{i}\right\} \equiv \boldsymbol{p}$ and $\boldsymbol{P}(\boldsymbol{b})=$ $\left\{P\left(b_{j}\right)=q_{j}\right\} \equiv \boldsymbol{q}$, respectively, one decomposes the joint probabilities $\mathbf{P}(\boldsymbol{a} \wedge \boldsymbol{b})=\left\{P\left(a_{i} \wedge b_{j}\right)=\pi_{i, j}\right\} \equiv \pi$ of the simultaneous events $\boldsymbol{a} \wedge \boldsymbol{b}$, as products $\left\{\pi_{i, j}=p_{i} P(j \mid i)\right\}$ of the marginal probability $p_{i}=P\left(a_{i}\right)$ of $i$ th event in $\boldsymbol{a}$ and the conditional probability $P(j \mid i)=P\left(a_{i} \wedge b_{j}\right) / P\left(a_{i}\right)$ of the event $b_{j}$ of $\boldsymbol{b}$, given that the event $a_{i}$ has already occurred:

$$
\begin{gathered}
\sum_{j} \pi_{i, j}=p_{i}, \quad \sum_{i} \pi_{i, j}=q_{j}, \quad \sum_{i} \sum_{j} \pi_{i, j}=1 ; \\
\sum_{j} P(j \mid i)=1, \quad i=1,2, \ldots, n .
\end{gathered}
$$

In this two-scheme scenario, the Shannon entropy of the product distribution $\pi$ (Fig. 1)

$S(\boldsymbol{\pi})=S(\boldsymbol{p})+\sum_{i} p_{i} S(q \mid i) \equiv S(\mathrm{~A})+S(\mathrm{~B} \mid \mathrm{A})$,

is seen to be given by the sum of the average entropy $S(\mathrm{~A})$ in the marginal probability distribution and the average conditional entropy $S(\mathrm{~B} \mid \mathrm{A})$ in $\boldsymbol{q}$ given $\boldsymbol{p}$ :

$$
\begin{aligned}
S(\mathrm{~B} \mid \mathrm{A}) & \left.=S(\boldsymbol{q} \mid \boldsymbol{p})=-\sum_{i} \sum_{j} \pi_{i, j} \log P(j \mid i)\right] \\
& =-\sum_{i} p_{i}\left[\sum_{j} P(j \mid i) \log P(j \mid i)\right] \equiv \sum_{i} p_{i} S(\boldsymbol{q} \mid i) .
\end{aligned}
$$

The latter represents the extra amount of uncertainty about the occurrence of outcomes $\boldsymbol{b}$, given that the events $\boldsymbol{a}$ are known to have occurred.

In other words, the amount of information obtained as a result of simultaneously observing the events $\boldsymbol{a}$ and 
Fig. 1 Entropy diagrams for the two (upper part) and three (lower part) dependent probability schemes
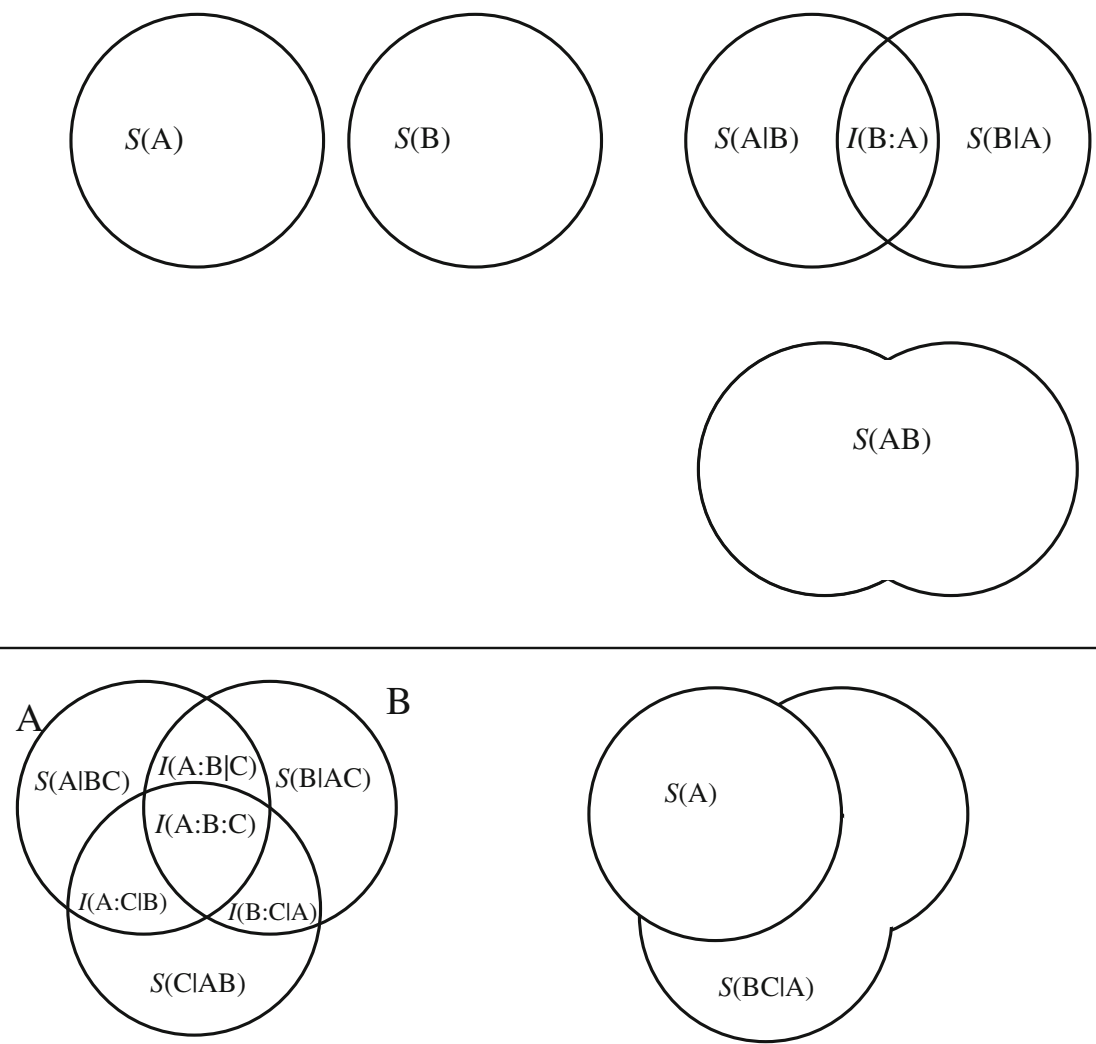

$\mathrm{C}$
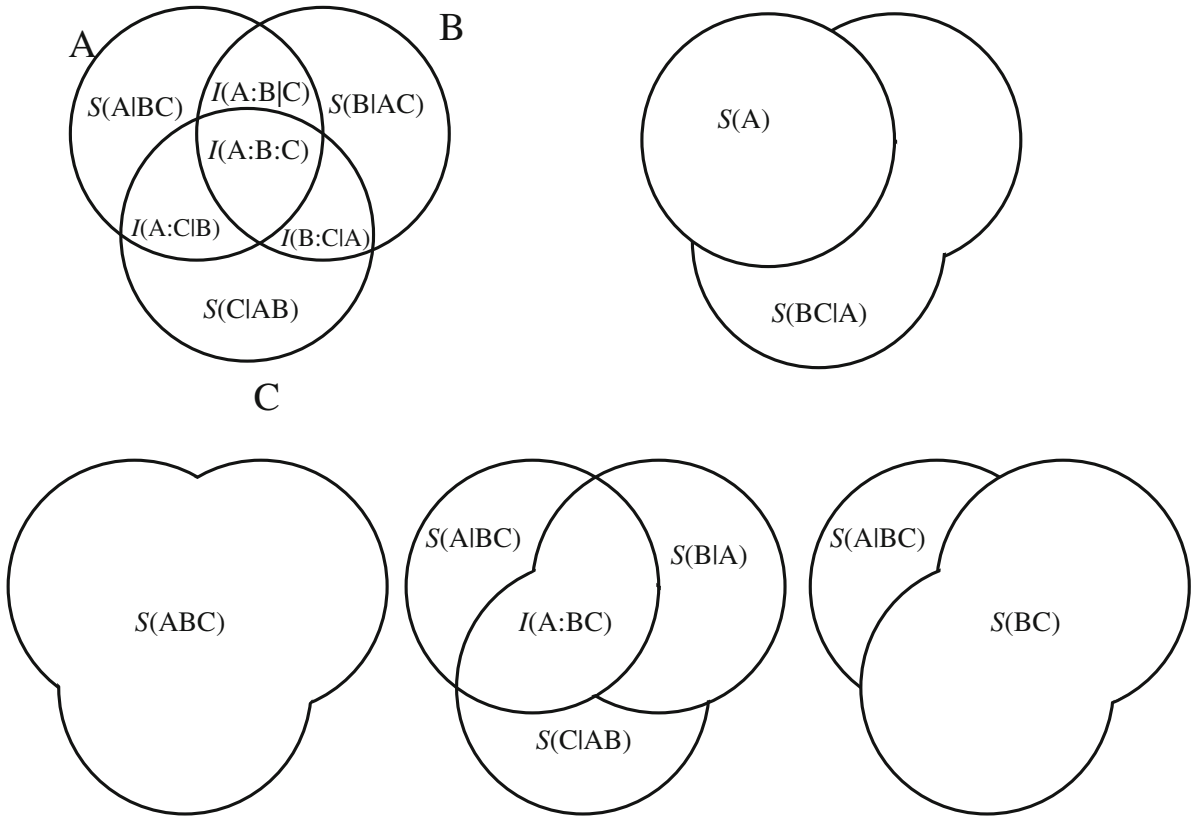

$\boldsymbol{b}$ of the two discrete probability distributions $\boldsymbol{p}=\boldsymbol{P}(\boldsymbol{a})$ and $\boldsymbol{q}=\boldsymbol{P}(\boldsymbol{b})$, respectively, equals the amount of information observed in set $\boldsymbol{a}$, supplemented by the extra information provided by the occurrence of events in the other set $\boldsymbol{b}$, when $\boldsymbol{a}$ are known to have occurred already.

Clearly, by using the other probability distribution $\boldsymbol{q}=\left\{P\left(b_{i}\right)\right\}$ as the marginal one, one arrives at the alternative expression for $S(\pi)$ :

$S(\boldsymbol{\pi})=S(\boldsymbol{q})+\sum_{j} q_{j} S(p \mid j) \equiv S(\mathrm{~B})+S(\mathrm{~A} \mid \mathrm{B})$.

One similarly defines the average mutual information $I(\mathrm{~A}: \mathrm{B})$ in these two probability distributions (see Fig. 1):

$$
\begin{aligned}
I(\mathrm{~A}: \mathrm{B}) & =I(\boldsymbol{p}: \boldsymbol{q})=S(\mathrm{~A})-S(\mathrm{~A} \mid \mathrm{B}) \\
& =S(\mathrm{~B})-S(\mathrm{~B} \mid \mathrm{A})=S(\mathrm{~A})+S(\mathrm{~B})-S(\mathrm{AB}) \\
& =\sum_{i} \sum_{j} \pi_{i, j} \log \left(\pi_{i, j} / \pi_{i, j}^{0}\right)=S\left(\pi^{0}\right)-S(\boldsymbol{\pi}) \\
& =S(\boldsymbol{p})+S(\boldsymbol{q})-S(\boldsymbol{\pi})=S(\boldsymbol{p})-S(\boldsymbol{p} \mid q) \\
& =S(\boldsymbol{q})-S(\boldsymbol{q} \mid \boldsymbol{p}) \geq 0 .
\end{aligned}
$$

where $\pi^{0}=\left\{\pi_{i, j}^{0} \equiv p_{i} q_{j}\right\}$. Thus, this average mutual information represents the entropy deficiency measuring the missing information between the joint probabilities $\mathbf{P}(\boldsymbol{a} \wedge \boldsymbol{b})=\boldsymbol{\pi}$ of the dependent events $\boldsymbol{a}$ and $\boldsymbol{b}$, and the joint probabilities $\mathbf{P}^{\text {ind. }}(\boldsymbol{a} \wedge \boldsymbol{b})=\pi^{0} \equiv \boldsymbol{p} \otimes \boldsymbol{q}$ for the independent events: $I(\boldsymbol{p}: \boldsymbol{q})=\Delta S\left(\pi \mid \pi^{0}\right)$. A similar information-distance interpretation can be attributed to the conditional entropy: $S(\boldsymbol{p} \mid \boldsymbol{q})=S(\boldsymbol{p})-\Delta S\left(\pi \mid \pi^{0}\right)$. 
Fig. 2 Communication channel of the 2-AO model of the chemical bond and its entropy/ information descriptors (in bits)

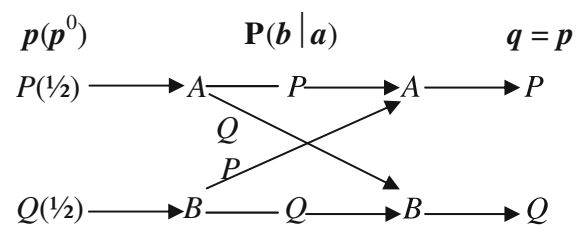

The entropy/information descriptors of three-dependent probability schemes $\mathrm{A}, \mathrm{B}$ and $\mathrm{C}$ are summarized in the lower part of Fig. 1. They are required to describe the many-orbital effects, e.g., the coupling between chemical bonds, molecular sites, and reactants [2, 3, 31, 32] (see the "Ionic promotion of reactants by a catalyst" section).

We conclude this short overview with the key entropy/ information descriptors of the information propagation in the communication system [1, 7-9]. In such a "device" (see Fig. 2), the input signal emitted from $n$ "inputs" $\boldsymbol{a}=\left(a_{1}\right.$, $a_{2}, \ldots, a_{n}$ ) of the channel source (A) is characterized by the input probability distribution $\boldsymbol{P}(\boldsymbol{a})=\boldsymbol{p}=\left(p_{1}, p_{2}, \ldots\right.$, $\left.p_{n}\right) \equiv \boldsymbol{P}(\mathrm{A})$. It can be received at $m$ "outputs" $\boldsymbol{b}=\left(b_{1}, b_{2}\right.$, $\ldots, b_{m}$ ) of the system receiver (B). The distribution of the output signal among the detection "events" $\boldsymbol{b}$ gives rise to the output probability distribution $\boldsymbol{P}(\boldsymbol{b})=\boldsymbol{q}=\left(q_{1}, q_{2}, \ldots\right.$, $\left.q_{m}\right) \equiv \boldsymbol{P}(\mathrm{B})$. The transmission of signals is randomly disturbed within the communication system, thus exhibiting a typical communication noise. This is because the signal sent at the given input is in general received with a nonzero probability at several outputs. This feature of communication systems is described by the spread in the conditional probabilities of the outputs-given-inputs, $\mathbf{P}(\mathrm{B} \mid \mathrm{A})=$ $\left\{P\left(b_{j} \mid a_{i}\right)=P\left(a_{i} \wedge b_{j}\right) / P\left(a_{i}\right) \equiv P(j \mid i)\right\}$, where $P\left(a_{i} \wedge b_{j}\right) \equiv \pi_{i, j}$ stands for the probability of the joint-occurrence of the specified pair of the input-output events.

The average conditional entropy $S(\mathrm{~B} \mid \mathrm{A}) \equiv S(\boldsymbol{q} \mid \boldsymbol{p})$ is thus determined by the scattering probabilities $\mathbf{P}(\mathrm{B} \mid \mathrm{A})=$ $\left\{P\left(b_{j} \mid a_{i}\right) \equiv P(j \mid i)\right\} \equiv \mathbf{P}(\boldsymbol{b} \mid \boldsymbol{a})$. This entropy measures the average noise in the transmission of signals, while the average amount of information flowing through the channel is measured by the mutual information in the source and receiver probability distributions $I(\mathrm{~A}: \mathrm{B})=$ $S(\boldsymbol{p})-S(\boldsymbol{p} \mid \boldsymbol{q}) \equiv I(\boldsymbol{p}: \boldsymbol{q})$. The latter reflects the net amount of information transmitted through the communication channel, while the conditional information $S(p \mid q) \equiv$ $S(\mathrm{~A} \mid \mathrm{B})$ reflects in the channel "noise" part of $S(\boldsymbol{p})=$ $S(\boldsymbol{p} \mid \boldsymbol{q})+I(\boldsymbol{p}: \boldsymbol{q})$. Accordingly, $S(\boldsymbol{q} \mid \boldsymbol{p}) \equiv S(\mathrm{~B} \mid \mathrm{A})$ reflects the noise part of $S(\boldsymbol{q}): S(\boldsymbol{q})=S(\boldsymbol{q} \mid \boldsymbol{p})+I(\boldsymbol{p}: \boldsymbol{q})$.

\section{Bond components in 2-AO model}

Consider now the familiar problem of combining the two (Löwdin-orthogonalized) $\mathrm{AO}, A(\boldsymbol{r})$ and $B(\boldsymbol{r})$, say, two $1 s$ orbitals centered on nuclei A and $\mathrm{B}$, respectively, which contribute a single electron each to form the chemical bond
A-B. The two basis functions $\chi=(A, B)$ then form the bonding $\left(\varphi_{\mathrm{b}}\right)$ and anti-bonding $\left(\varphi_{\mathrm{a}}\right)$ MO combinations, $\boldsymbol{\varphi}=\left(\varphi_{\mathrm{b}}, \varphi_{\mathrm{a}}\right)=\chi \mathbf{C}:$

$\varphi_{\mathrm{b}}=\sqrt{P} A+\sqrt{Q} B, \quad \varphi_{\mathrm{a}}=-\sqrt{Q} A+\sqrt{P} B$,

$P+Q=1$,

where the square matrix

$\mathbf{C}=\left[\begin{array}{cc}\sqrt{P} & -\sqrt{Q} \\ \sqrt{Q} & \sqrt{P}\end{array}\right]=\left[\boldsymbol{C}_{\mathrm{b}} \mid \boldsymbol{C}_{\mathrm{a}}\right]$

groups the LCAO MO expansion coefficients, expressed here in terms of the complementary probabilities $P$ and $Q=1-P$. The former marks the conditional probabilities

$P\left(A \mid \varphi_{\mathrm{b}}\right)=\left|C_{A, \mathrm{~b}}\right|^{2}=P\left(B \mid \varphi_{\mathrm{a}}\right)=\left|C_{B, \mathrm{a}}\right|^{2} \equiv P$,

and the latter measures the remaining matrix elements of the conditional probability matrix, of observing $\mathrm{AO}$ in $\mathrm{MO}$ :

$P\left(B \mid \varphi_{\mathrm{b}}\right)=\left|C_{B, \mathrm{~b}}\right|^{2}=P\left(A \mid \varphi_{\mathrm{a}}\right)=\left|C_{A, \mathrm{a}}\right|^{2}=Q$.

In OCT, the ground-state configuration of the doubly occupied bonding $\mathrm{MO} \varphi_{\mathrm{b}}$, for which the model charge-andbond-order (CBO) matrix, the double density matrix $\mathbf{d}$,

$$
\begin{aligned}
\gamma & =\left\{\gamma_{i, j}\right\}=2\left\langle\chi \mid \varphi_{\mathrm{b}}\right\rangle\left\langle\varphi_{\mathrm{b}} \mid \boldsymbol{\chi}\right\rangle=2 \mathbf{d}=2 \mathbf{C}_{\mathrm{b}} \mathbf{C}_{\mathrm{b}}^{\dagger} \\
& =2\left[\begin{array}{cc}
P & \sqrt{P Q} \\
\sqrt{Q P} & Q
\end{array}\right],
\end{aligned}
$$

generates the following conditional probability matrix of $\mathrm{AO}$ in the molecular bond system $[2,3,26]$ :

$\mathbf{P}(\boldsymbol{b} \mid \boldsymbol{a})=\mathbf{P}(\boldsymbol{\chi} \mid \boldsymbol{\chi})=\left\{P(j \mid i)=\gamma_{i, j} \gamma_{j, i} /\left(2 \gamma_{i, i}\right)\right\}=\left[\begin{array}{ll}P & Q \\ P & Q\end{array}\right]$.

It determines the relevant $\mathrm{AO} \rightarrow \mathrm{AO}$ communication network for this model bond system shown in Fig. 2. In this non-symmetrical binary channel, one adopts the molecular input signal, $\boldsymbol{p}=(P, Q)$, to extract the bond IT-covalency index, which measures the channel average communication noise, and the promolecular input reference $\boldsymbol{p}^{0}$, in which the two basis functions contribute a single electron each to form the chemical bond, $\boldsymbol{p}^{0}=(1 / 2,1 / 2)$, to calculate the ITionicity index measuring the channel information capacity $[1-3,32]$. The bond IT-covalency $N_{\mathrm{S}}^{\text {cov }}(P)=S(\boldsymbol{q}=\boldsymbol{p} \mid \boldsymbol{p})$ is thus determined by the binary entropy function $H(P)=$ - $P \log _{2} P-Q \log _{2} Q=H(\boldsymbol{p})$ (see Figs. 2, 3), reaching the maximum value $H(P=1 / 2)=1$ bit for the symmetric bond 


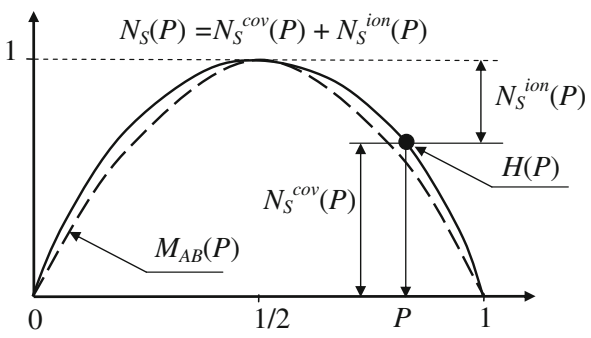

Fig. 3 Conservation of the overall entropic bond multiplicity $N_{\mathrm{S}}(P)=1$ bit in the 2 -AO model of the chemical bond, combining the conditional entropy (average noise, covalency) $N_{\mathrm{S}}^{\operatorname{cov}}(P)=$ $H(P)$ and the mutual information (information capacity, ionicity) $N_{\mathrm{S}}^{\text {cov }}(P)=1-H(P)$. In MO theory the direct bond-order of Wiberg is represented by (broken line) parabola $M_{A, B}(P)=4 P(1-P) \equiv$ $4 P Q=V^{\mathrm{cov}}(P)+V_{\mathrm{oc}}^{\mathrm{ion}}(P) \equiv V(P)$, which in the quadratic difference approach separates the two-centre ionicity $V_{\mathrm{tc}}^{\mathrm{ion}}(P)=1-V(P)$ (upper area above the parabola) from the sum $V(P)$ of the overall covalent component $V^{\mathrm{cov}}(P)$ and the one-centre ionicity $V_{\mathrm{oc}}^{\text {ion }}(P)$

$P=Q=1 / 2$, e.g., the $\sigma$ bond in $\mathrm{H}_{2}$ or the $\pi$-bond in ethylene, and vanishes for the lone-pair molecular configurations, when $P=(0,1), H(P=0)=H(P=1)=0$, marking the alternative ion-pair configurations $\mathrm{A}^{+} \mathrm{B}^{-}$and $\mathrm{A}^{-} \mathrm{B}^{+}$, respectively, relative to the initial $\mathrm{AO}$ occupations $N^{0}=(1,1)$ in the assumed (atomic) promolecular reference, in which both atoms contribute a single electron each to form the chemical bond.

The complementary descriptor $N_{\mathrm{S}}^{\text {ion }}(P)=I\left(\boldsymbol{p}^{0}: \boldsymbol{p}\right)=$ $\Delta S\left(\boldsymbol{p} \mid \boldsymbol{p}^{0}\right)+S(\boldsymbol{p})-S(\boldsymbol{p} \mid \boldsymbol{p}) \equiv I^{0}(P)$ of the model IT-ionicity, also plotted in Fig. 3, which determines the channel mutual information (capacity), $I^{0}(P)=H\left[p^{0}\right]-H(P)=$ $1-H(P)$, reaches the highest value for these two limiting electron-transfer configurations $P=(0,1): I^{0}(P=0)=$ $I^{0}(P=1)=H(1 / 2)=1 \mathrm{bit}$, and identically vanishes for the purely covalent, symmetric bond, $I^{0}(P=1 / 2)=0$. As explicitly shown in Fig. 3, these two components of the chemical bond multiplicity compete with one another, yielding the conserved overall IT bond index $N_{\mathrm{S}}(P)=$ $N_{\mathrm{S}}^{\mathrm{cov}}(P)+N_{\mathrm{S}}^{\text {ion }}(P)=H\left[\boldsymbol{p}^{0}\right]=1$ bit, marking a single bond in IT in the whole range of the admissible bond polarizations $P \in[0,1]$.

This simple model thus properly accounts for the competition between the bond covalency and ionicity, while preserving the single bond-order measure reflected by the conserved overall IT multiplicity of the chemical bond. Similar effects transpire from this model description in CTCB and from the quadratic bond indices formulated in the MO theory [41, 42]. The Wiberg bond-order [37] plot for this model is given by the parabola displayed in Fig. 3,

$M_{A, B}(P)=\left[\gamma_{A, B}(P)\right]^{2}=4 P Q=4 P(1-P)$,

which closely resembles the IT-covalent plot $N_{\mathrm{S}}^{\mathrm{cov}}(P)=$ $H(P)$ in the figure.

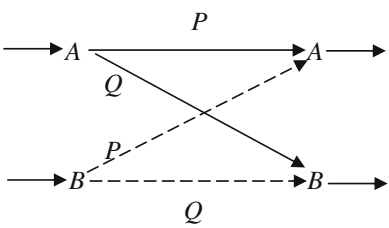

Fig. 4 The elementary (row) sub-channels due to inputs A (solid lines) and $\mathrm{B}$ (broken lines) in the 2-AO model of the chemical bond. $S_{\mathrm{AB}}\left(\chi_{\mathrm{AB}} \mid A\right)=S_{\mathrm{AB}}\left(\chi_{\mathrm{AB}} \mid B\right)=H(P), I_{\mathrm{AB}}^{0}\left(A: \chi_{\mathrm{AB}}\right)=I_{\mathrm{AB}}^{0}\left(B: \chi_{\mathrm{AB}}\right)=$ $1-H(P)$

The bond components of Fig. 3 can be further decomposed into the corresponding atomic bond contributions. In the communication theory this partition is accomplished by using the partial (row) sub-channels of Fig. 4, each determining communications for the specified atomic input $[1,54,55]$.

Figure 5a reflects a competition between two atoms for the bonding (shared) electrons, with the maximum $S_{A}^{r}\left(P_{A}^{\max }\right)$ $\left[S_{B}^{r}\left(P_{B}^{\max }\right)\right]$ being observed for $P_{A}^{\max }>1 / 2\left(P_{B}^{\max }<1 / 2\right)$. It follows from this atomic perspective that the maximum molecular entropy-covalency $H(P)$, for $P=Q=1 / 2$, marks the "compromise" between the corresponding atomic contributions $S_{A}^{r}(1 / 2)=S_{B}^{r}(1 / 2)=1 / 2$ bit, for which the overall covalency exactly exhausts the total IT bond-order $H(1 / 2)=$ $N^{r}(P)=1$ bit, thus marking the purely covalent bond: $I^{r}(1 / 2)=0$. A similar competition between atoms can be detected in the atomic ionic indices, which reach the highest values $I_{A}^{r}(1)=I_{B}^{r}(0)=1$ bit when the two electrons occupy the same AO. Indeed, these extreme MO polarizations mark the lone electron pair configurations, i.e., the ion-pairs $\mathrm{A}^{-} \mathrm{B}^{+}$ or $\mathrm{A}^{+} \mathrm{B}^{-}$bonded by the single, purely ionic bond, for which all covalent contributions in the model exactly vanish.

It should be observed that the atomic ionic index $I_{B}^{r}(P)$ reaches the minimum (negative) value at a slightly higher value of $P$ compared to $P_{A}^{\max }$, which also gives rise to the negative minimum feature of $N_{B}^{r}(P)$ in this region, where the covalency index of the other atom, $S_{A}^{r}(P)$, reaches the maximum value. The negative mutual information value reflects the donor-acceptor (coordination) interaction $B \rightarrow A$ in this region of the MO polarization parameter $P$ : $1 / 2<P<1$. A similar, complementary behavior is observed in $I_{A}^{r}(P)$ and the associated overall index $N_{A}^{r}(P)$, with both exhibiting negative-valued minima for $0<P<1 / 2$, where $S_{B}^{r}(P)$ reaches the maximum value. The partial row-channels thus generate an attractive perspective on the atomic contributions to IT bond-orders. They generate the exhaustive probability-partitioning scheme, for dividing the diatomic chemical bond descriptors into atomic contributions. The diagram of Fig. 5a also generates a framework for estimating relative roles played by specific bond components for different MO polarizations.

In typical SCF LCAO MO calculations the lone pairs of the valence and/or inner-shell electrons can strongly affect the overall IT descriptors of the chemical bonds. 
(a)

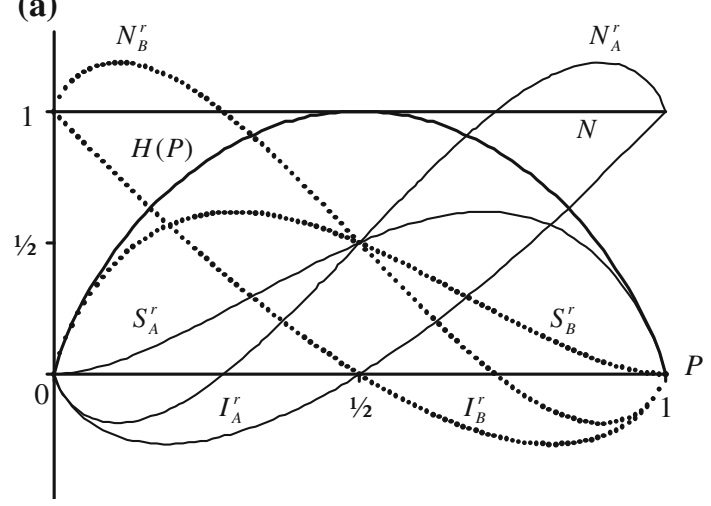

(b)

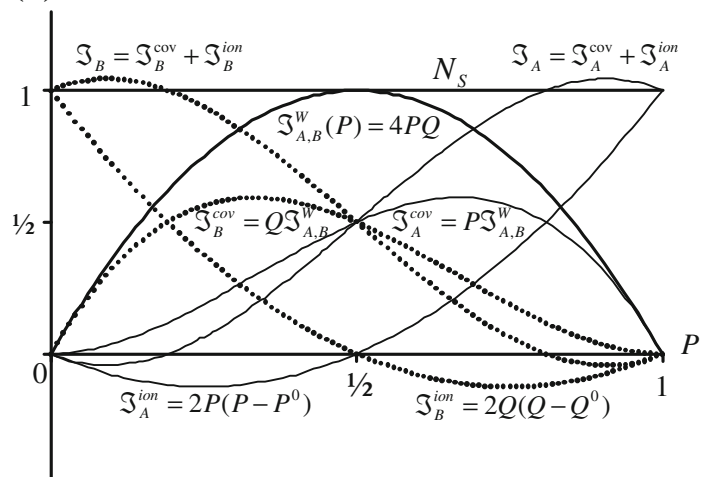

Fig. 5 Atomic bond contributions from the Communication (a) and MO (b) theories. The entropy-covalency $S_{X}^{r}$, information-ionicity $I_{X}^{r}$, and overall atomic bond index $N_{X}^{r}=S_{X}^{r}+I_{X}^{r}$ (in bits) in (a) describe the atomic row-channels of Fig. 4, while the corresponding MO indices $\Im_{X}^{\text {cov }}$, $\Im_{X}^{\text {ion }}, \Im_{X}=\Im_{X}^{\text {cov }}+\Im_{X}^{\text {ion }}$ in (b) represent the atomic MO covalencies, ionicities and overall bond indices, respectively. The covalent contributions are from the probability-partitioning of the Wiberg index $\Im_{A, B}^{\mathrm{W}}=4 P Q \equiv M_{A B}$, while the atomic ionicities represent the probability weighted charge displacements: $\Im_{X}^{\text {ion }}=P_{X} \Delta q_{X}=2 P_{X} \Delta P_{X}$. The overall bond-orders in (a): $S^{r}(P)=S_{A}^{r}(P)+S_{B}^{r}(P)=H(P), I^{r}(P)=$ $I_{A}^{r}(P)+I_{B}^{r}(P)=1-H(P)$, and the conserved total bond multiplicity, $N=S^{r}(P)+I^{r}(P)=1$ bit, are also plotted. In (b) the overall covalency $\Im^{\text {cov }}(P)=\Im_{A}^{\text {cov }}(P)+\Im_{B}^{\text {cov }}(P)=\Im_{A, B}^{\mathrm{W}}(P)=4 P Q$, and the conserved total bond-order $N_{\mathrm{S}}=\Im^{\operatorname{cov}}(P)+\left[\Im_{A}^{\text {ion }}(P)+\Im_{B}^{\text {ion }}(P)\right] \equiv \Im^{\text {cov }}(P)+$ $\Im^{\text {ion }}(P)=1$, are also shown

Elimination of such lone-pair contributions to the resultant IT bond indices of diatomic fragments in molecules requires an ensemble (flexible input) approach [2, 3, 33, 34], in which the input probabilities are derived from the joint (bond) probabilities of two AO centered on different atoms, which reflect the simultaneous participation of the given pair of basis functions in the inter-atomic chemical bonds. Such an approach effectively projects out the spurious contributions due to the inner- and outer-shell AO, which are excluded from mixing into the delocalized, bonding MO combinations. This probability-weighting procedure is capable of reproducing the Wiberg bond-order in diatomics, at the same time providing the IT-covalent/ ionic resolution of this overall bond index.

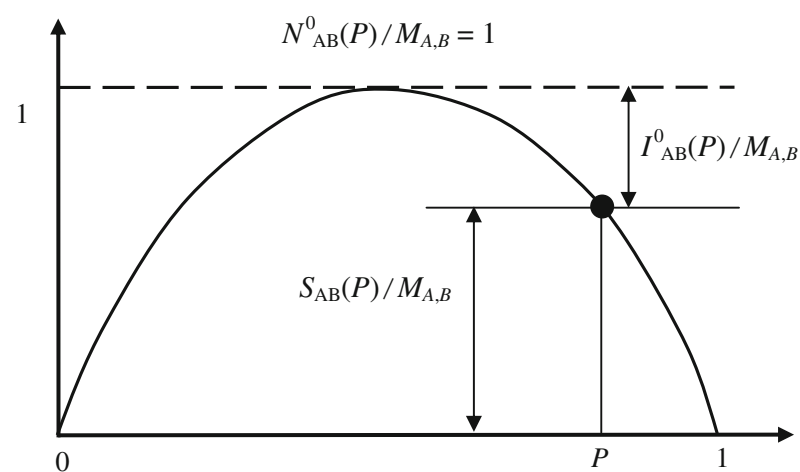

Fig. 6 Variations of the IT-covalent $\left[S_{\mathrm{AB}}(P)\right]$ and and IT-ionic $\left[I_{\mathrm{AB}}^{0}(P)\right]$ components (in $M_{A, B}$ units) of the chemical bond in the 2-AO model (see Figs. 2, 3) with changing MO polarization $P$ and the conservation of the relative total bond-order $N_{\mathrm{AB}}^{0}(P) / M_{A, B}=1$

Let us illustrate this weighting procedure in 2-AO model. In the bond-weighted approach one again uses the elementary sub-channels of Fig. 4 and their entropy/ information descriptors. The conditional entropy and mutual information quantities for these partial communication systems, $\left.\left\{S_{\mathrm{AB}}\left(\chi_{\mathrm{AB}} \mid i\right)\right\}, I_{\mathrm{AB}}^{0}\left(i: \chi_{\mathrm{AB}}\right) ; i=A, B\right\}$ are also listed in the diagram. Since the row-descriptors represent the IT indices per electron in the diatomic fragment containing $N_{\mathrm{AB}}$ electrons, these contributions have to be multiplied by $N_{\mathrm{AB}}=N=2$ in the corresponding resultant covalent/ionic and overall measures.

Therefore, using the off-diagonal joint probability $P(A \wedge B)=P(B \wedge A)=P Q=\gamma_{A, B} \gamma_{B, A} / 4$ as the ensemble probability for both AO inputs gives the following average quantities for this model diatomic system:

$$
\begin{aligned}
S_{\mathrm{AB}} & =N_{\mathrm{AB}}\left[P(A \wedge B) S_{\mathrm{AB}}\left(\chi_{\mathrm{AB}} \mid A\right)+P(B \wedge A) S_{\mathrm{AB}}\left(\chi_{\mathrm{AB}} \mid B\right)\right] \\
& =4 P Q H(P)=M_{A, B} H(P), \\
I_{\mathrm{AB}}^{0} & =N_{\mathrm{AB}}\left[P(A \wedge B) I_{\mathrm{AB}}^{0}\left(A: \chi_{\mathrm{AB}}\right)+P(B \wedge A) I_{\mathrm{AB}}^{0}\left(B: \chi_{\mathrm{AB}}\right)\right] \\
& =4 P Q[1-H(P)]=M_{A, B}[1-H(P)], \\
N_{\mathrm{AB}}^{0} & =S_{\mathrm{AB}}+I_{\mathrm{AB}}^{0}=4 P Q=\left(\gamma_{A, B}\right)^{2}=M_{A, B} .
\end{aligned}
$$

We have thus recovered the Wiberg index as the overall IT descriptor of the chemical bond in 2-AO model, at the same time establishing its covalent, $S_{\mathrm{AB}}$, and ionic, $I_{\mathrm{AB}}^{0}$, contributions. It follows from Fig. 6 that these IT-covalency and IT-ionicity components compete with one another while conserving the Wiberg bond-order as the overall information measure of the bond multiplicity (in bits) for this model.

\section{Local probes of molecular electron distributions}

The densities of the average information measures have also been used as efficient probes of the chemical bond 
(a)
[1.1.1]

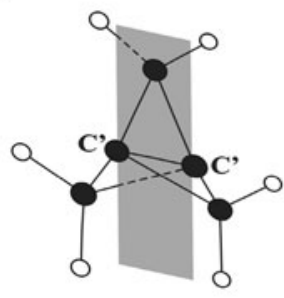

(c)

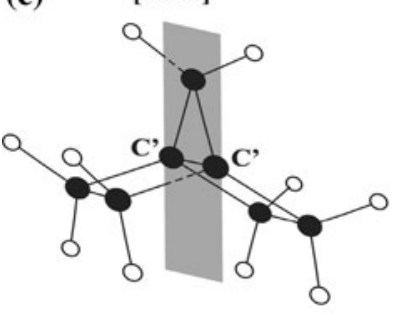

(b)

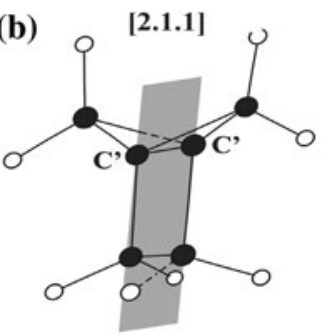

(d)

[2.2.2]

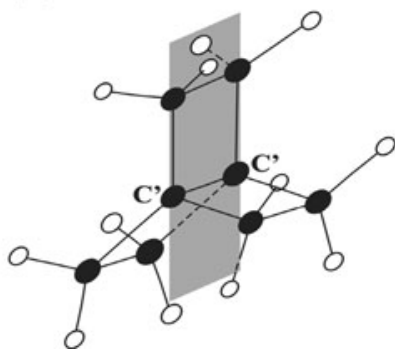

Fig. 7 The propellane structures and the planes of sections containing the bridge and bridgehead $\left(\mathrm{C}^{\prime}\right)$ carbon atoms, identified by black circles

patterns in molecules. One of the challenging problems is the nature of the central bond in small propellanes between the bridgehead carbons (Fig. 7) [1-4, 17, 18]. It can be diagnosed using the density difference $\Delta \rho(\boldsymbol{r})$ function, which has traditionally been employed to extract the electron redistributions accompanying the bond formation. Alternatively, it can be investigated with the help of appropriate local IT probes [1, 4], e.g., the entropy-deficiency density $\Delta s(\boldsymbol{r})$ relative to the promolecular distribution $\rho^{0}(\boldsymbol{r})$,

$\Delta s(\boldsymbol{r})=\rho(\boldsymbol{r}) \log \left[\rho(\boldsymbol{r}) / \rho^{0}(\boldsymbol{r})\right]$,

or it can be diagnosed using the local displacement in the Shannon entropy:

$\Delta h(\boldsymbol{r})=-\rho(\boldsymbol{r}) \log \rho(\boldsymbol{r})+\rho^{0}(\boldsymbol{r}) \log \rho^{0}(\boldsymbol{r})$.

Figure 8 compares these local probes for the central and carbon-bridge bonds in the propellanes of Fig. 7 [4]. The density difference plots show that in the small [1.1.1] and [2.1.1] systems there is on average a depletion of the electron density, relative to promolecule, between the bridgehead carbon atoms, while larger [2.2.1] and [2.2.2] systems exhibit a net density buildup in this region. A similar conclusion follows from the entropy-displacement and entropy-deficiency plots of the figure, as well as from the CG analysis using the Fisher measure of information (see the "CG criterion of bond localization" section) $[2,21]$.

The reason for this apparent absence of the direct chemical interaction between bridgehead carbons in the [1.1.1] propellane and its presence in the [2.2.2] system is examined in the simple localized perspective on the central and bridge bonds between carbon atoms shown in Fig. 9 $[3,18]$. As argued in the figure, the minimum (valence) basis set description of the bond structure in these two systems can be qualitatively understood in terms of the localized MO resulting from interactions between the directed (hybrid) orbitals on neighboring atoms, and the non-bonding electrons occupying the non-overlapping atomic hybrids.

In the smallest [1.1.1] system, the nearly tetrahedral $\left(h=s p^{3}\right.$ ) hybridization on both bridgehead and bridging carbons is required to form the chemical bonds of the three carbon bridges and to accommodate two hydrogens on each bridge-carbon. Thus, three $s p^{3}$ hybrid orbitals (HO) on each of the bridgehead atoms are used to form the chemical bonds with the bridge carbons and the fourth hybrid, directed away from the central-bond region, remains nonbonding and singly occupied.

In the large [2.2.2] propellane, the two central carbons acquire a nearly trigonal $\left(h^{\prime}=s p^{2}\right)$ hybridization, to form bonds with the bridge neighbors, each with a single $2 p$ orbital directed along the central-bond axis, which has not been used in this hybridization scheme, now being available to form a strong through-space component of the overall multiplicity of the $\mathrm{C}^{\prime}-\mathrm{C}^{\prime}$ bond. This difference in the promoted valence states of the central carbons in the two systems explains the missing direct component in the smaller (diradical) [1.1.1] propellane and its presence in the larger [2.2.2] system.

One thus concludes that small propellanes are not expected to exhibit the direct component of the chemical bond. The two entropic diagrams are seen to be qualitatively similar to the corresponding density difference plots. This resemblance is particularly strong between the $\Delta \rho(\boldsymbol{r})$ and $\Delta s(\boldsymbol{r})$ diagrams thus demonstrating that they represent equivalent local probes of the presence of the throughspace chemical bond(s).

However, as we shall argue in the "Indirect chemical interactions" section, besides this direct (through-space) bond component there also exist its indirect (throughbridge) complement, realized via carbon bridges, which explains the apparent existence of some central chemical bond even in the smallest propellane. In this more general outlook on the bond-order concept, emerging from both the Wiberg-type approach and OCT, the chemical bond measures a general "dependence" between projections of the basis functions onto the molecular bond-subspace spanned by all occupied MO. On one hand, this dependence between AO is partly realized directly (through space), by a constructive interference of orbitals (probability amplitudes) on two atoms involved, which increases the electron density between them. On the other hand, it has a partial indirect origin, through the orbitals of the remaining AIM. 
[1.1.1]
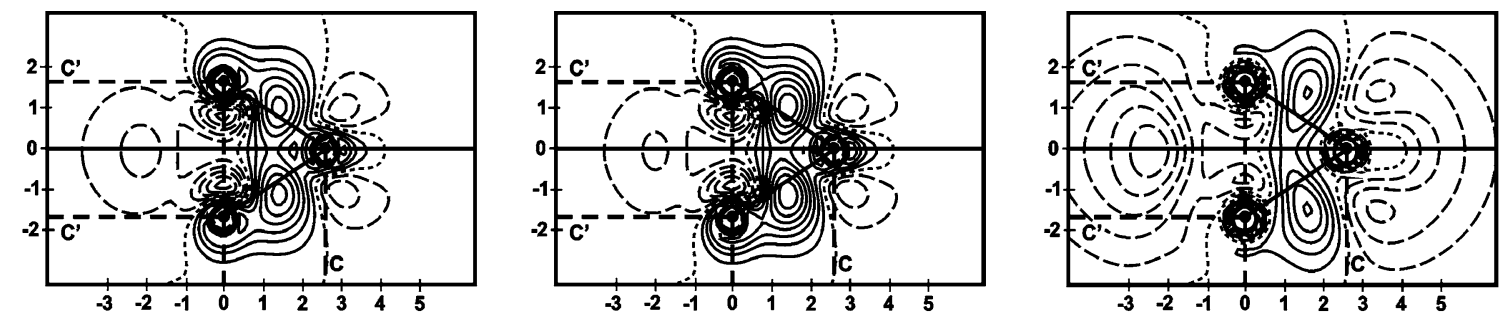

[2.1.1]
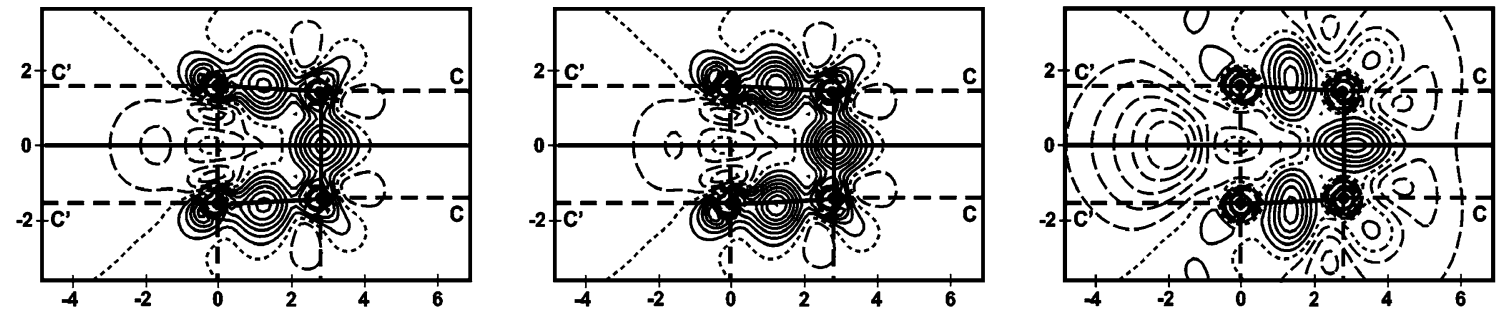

[2.2.1]
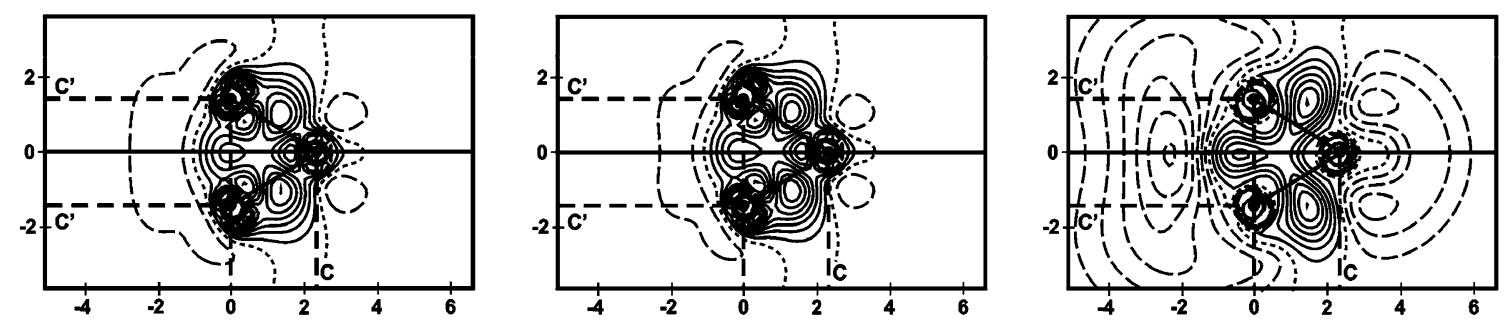

$[2.2 .2]$
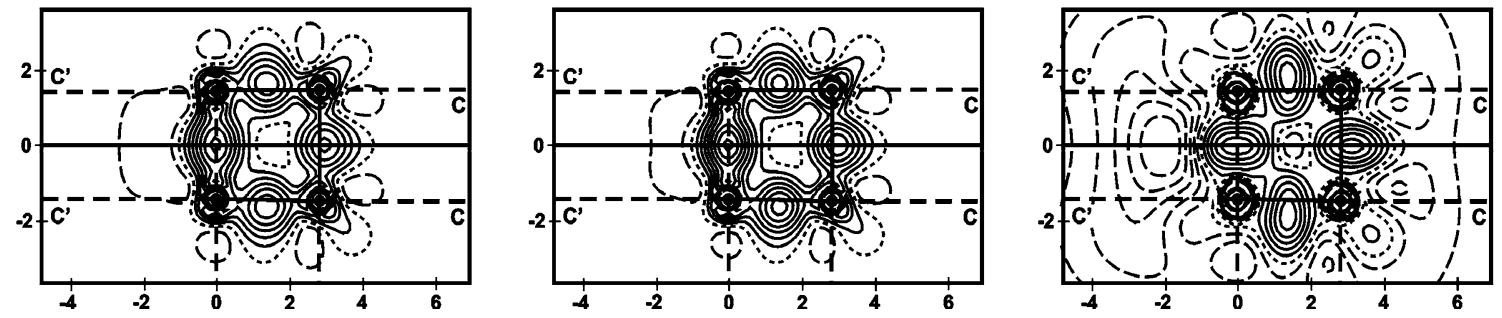

Fig. 8 A comparison between the equidistant contour maps of the density difference function $\Delta \rho(\boldsymbol{r})$ (first column), the informationdistance density $\Delta s(\boldsymbol{r})$ (second column), and the entropy-displacement

density $\Delta h(\boldsymbol{r})$ (third column), for the four propellanes of Fig. 7. The negative contour values are represented by broken lines

Fig. 9 Schematic diagrams of the localized bonds between carbon atoms in the [1.1.1] (a) and [2.2.2] (b) propellanes; the bridgehead carbon atoms are primed (a)

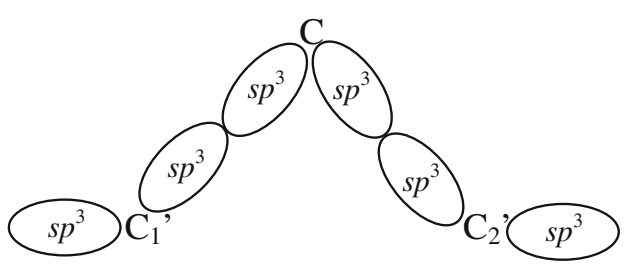

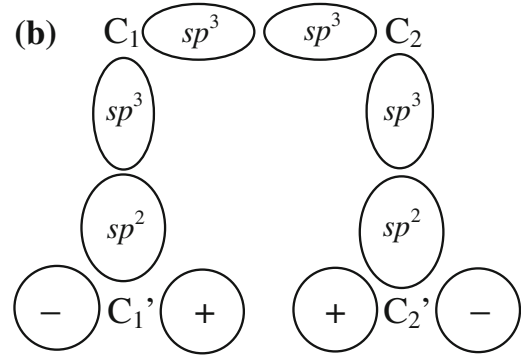

The latter is due to "geometrical" dependencies between the (non-orthogonal) projections of $\mathrm{AO}$ onto the bondsubspace of the molecule. The indirect chemical bonds are mainly realized through real chemical bridges in the molecule, e.g., the carbon bridges in propellanes. They effectively extend the range of chemical interactions in molecular systems (see the "Indirect chemical interactions" section). 


\section{CG criterion of bond localization}

Each scheme for resolving the molecular electron density, $\rho=\sum_{\alpha} \rho_{\alpha}$, into density pieces $\rho=\left\{\rho_{\alpha}\right\}$ of molecular fragments, e.g., the bonded atoms, MO or AO, implies the associated division of the total value of the molecular physical quantity $A$, represented by the functional $A[\rho] \equiv A^{\text {total }}[\rho]$, into the associated additive, $A^{\text {add. }}[\rho]$, and non-additive, $A^{\text {nadd. }}[\rho]$, contributions:

$$
\begin{gathered}
A[\rho] \equiv A^{\text {total }}[\boldsymbol{\rho}]=A^{\text {add. }}[\boldsymbol{\rho}]+A^{\text {nadd. }}[\boldsymbol{\rho}], \\
A^{\text {add. }}[\boldsymbol{\rho}] \equiv \sum_{\alpha} A\left[\rho_{\alpha}\right]
\end{gathered}
$$

where

$A^{\text {nadd }}[\boldsymbol{\rho}]=A^{\text {total }}[\boldsymbol{\rho}]-A^{\text {add. }}[\boldsymbol{\rho}]=A[\rho]-\sum_{\alpha} A\left[\rho_{\alpha}\right]$.

As indicated above, in the underlying multi-component system $A[\rho]$ becomes the functional of the whole vector of the subsystem densities, $A[\rho]=A^{\text {total }}[\boldsymbol{\rho}][1-3]$.

For example, this Gordon-Kim [56] division of the kinetic energy functional defines the non-additive contribution, which constitutes the basis of the DFT-embedding concept of Cortona and Wesołowski [57-63]. This division can be also used to partition the information quantities themselves [2, 3, 19-22, 26, 29]. In particular, the inverse of the non-additive Fisher information in the MO resolution [22] has been shown to define the IT-ELF concept [23-25], while the related quantity in the AO resolution [15] of the SCF MO theory offers the key CG criterion $[2,3,15,19-21]$ for localization of the chemical bonds in molecular systems.

In accordance with the prescription of Eq. (20), the nonadditive component of Fisher information (Eq. 2) in AO resolution $\left\{\chi_{\alpha}\right\}$, related to the associated non-additive part of the electronic kinetic energy, is defined by the difference between the total and additive information functionals:

$I^{\text {nadd. }}[\boldsymbol{\rho}]=I^{\text {total }}[\boldsymbol{\rho}]-I^{\text {add. }}[\boldsymbol{\rho}]=I[\rho]-\sum_{\alpha} I\left[\rho_{\alpha}\right]$

It has been argued elsewhere [2, 3, 15, 19-21] that the valence basins of its negative values efficiently locate the direct bonding regions in molecules, where the constructive interference of $\mathrm{AO}$ into the bonding $\mathrm{MO}$ takes place. Successful examples of such an analysis for butadiene and benzene, from SCF MO calculations in the minimum (STO-3G) basis set, are presented in the contour maps of Figs. 10 and 11. These diagrams testify to the efficiency of the $\mathrm{CG}$ criterion in localizing all $\mathrm{C}-\mathrm{C}$ and $\mathrm{C}-\mathrm{H}$ bonding regions in these two molecules. It indeed follows from the lower panels of Fig. 10, which explores the $\pi$ bonds in butadiene, that the peripheral neighboring carbons in butadiene are more strongly bonded than their central counterparts. The integration of such bonding basins of this information quantity provides the associated "occupation" indices [21] which can be used in a semiquantitative characterizations of such direct bonding interactions in molecular systems.

This novel visualization tool should prove useful in exploring the bonding patterns of controversial molecular systems, the bonding structure of which still remains a matter for scientific debate, with alternative bond criteria giving conflicting answers to the very qualitative question of the existence or non-existence of the disputed chemical bonds between the specified atoms in the molecular environment under consideration. A classical example is the central bond in small propellanes [2, 3, 19, 21], for which the VB-based charge-shift mechanism [17] has been proposed as an alternative explanation of its apparent existence.

\section{Indirect chemical interactions}

The chemical interaction between the specified pair of bonded atoms has recently been shown to exhibit both the direct (through-space) and, hitherto neglected, indirect (through-bridge) components resulting from the implicit dependencies between orbitals in the bond-subspace of the molecule [3, 20, 48-52]. The former reflects the direct interactions/communications between $\mathrm{AO}$, while the latter is realized indirectly, through the remaining basis functions which constitute an effective bridge for the implicit chemical coupling or the cascade probability scatterings between orbitals contributed by more distant atoms. The most efficient bridges for such an implicit bonding mechanism are real chemical bridges, originating from the basis functions contributed by the chemically bonded atoms connecting the given pair of the "terminal" AO in question. The through-bridge mechanism was demonstrated to result from the implicit dependencies between the (nonorthogonal) AO projections into the bonding subspace of the occupied MO [51, 52]. These AO components reflect their joint participation in the whole system of chemical bonds. The indirect, through-bridge mechanism thus represents a natural extension of the direct dependencies already manifested in the through-space bond components, e.g., those measured by the traditional Wiberg index.

In OCT the direct bond between the specified pair of interacting $\mathrm{AO}$, which constructively mix into the bonding MO, originates from their mutual probability scattering in the molecule. Its covalency represents a finite conditional probability due these molecular communications, related to the square of the corresponding element of the system $\mathrm{CBO}$ matrix, which couples the two basis functions, and hence also to the associated Wiberg bond-order contribution. 
Fig. 10 The contour diagrams of the CG density for butadiene: in the molecular plane (first panel) and in sections perpendicular to molecular plane along the chemical bond axes between the terminal (second panel) and the middle (third panel) carbon atoms $[19,21]$
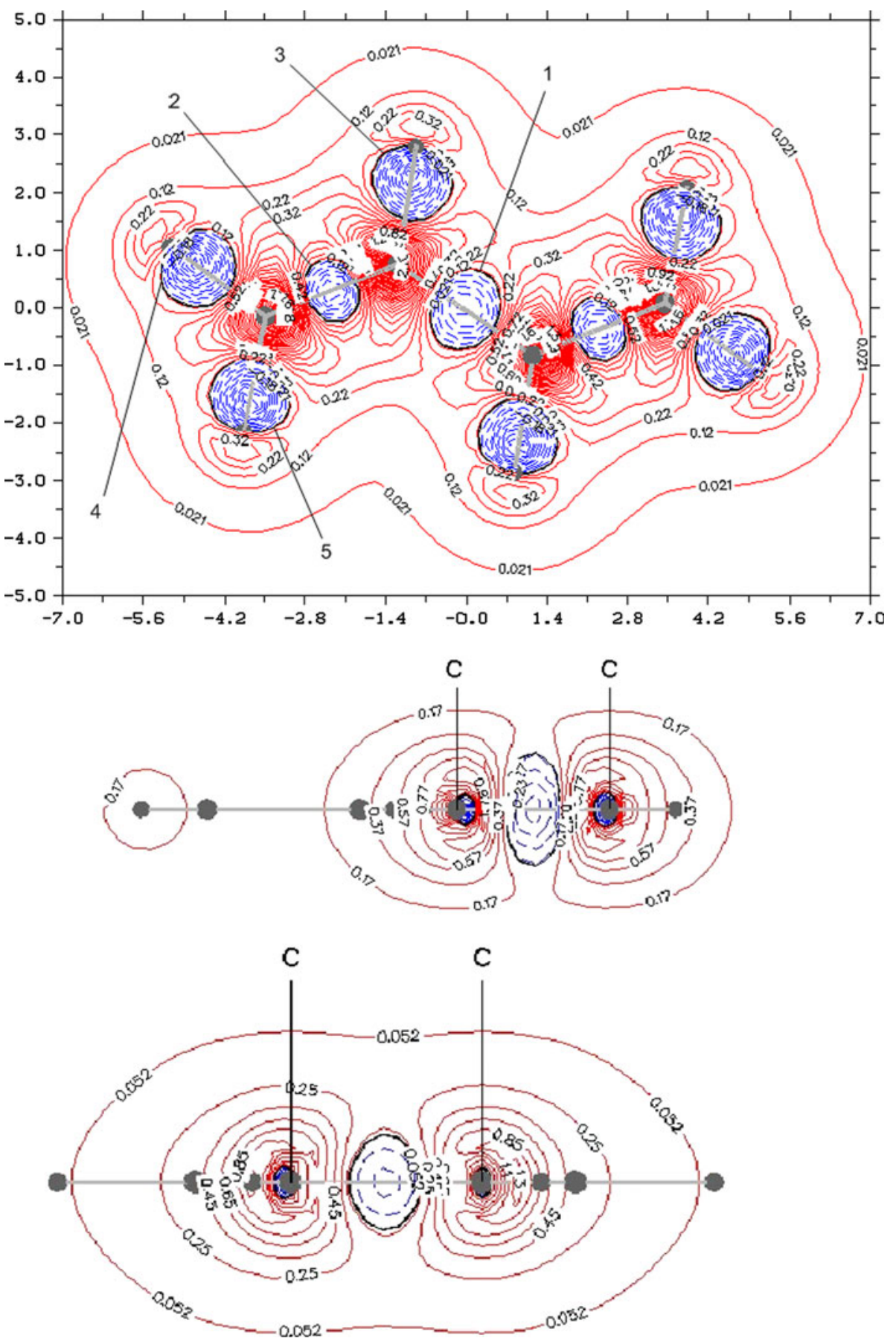

These direct $\mathrm{AO}$ communications are in accordance with the electron delocalization pattern implied by the system occupied (bonding) subspace of MO. The "implicit" (through-bridge) bond component can be similarly viewed as resulting from the indirect (cascade) information propagation via the bridging AO. Therefore, while the throughspace bonding reflects in OCT a direct "conversation" between $\mathrm{AO}$, the through-bridge bonding can be compared to a "hearsay" spreading between two AO in question via the connecting chain of orbital intermediaries involved in the effective communication chain under consideration.

One thus distinguishes in OCT the direct ("dialog") and indirect ("gossip") contributions to the effective IT bondorder, which together determine the resultant bond multiplicity between the given pair of AO. The direct (explicit) bonding interaction between neighboring atoms, reflected by the original Wiberg bond-orders, is generally associated with the presence of the bond-charge or an increase of the 

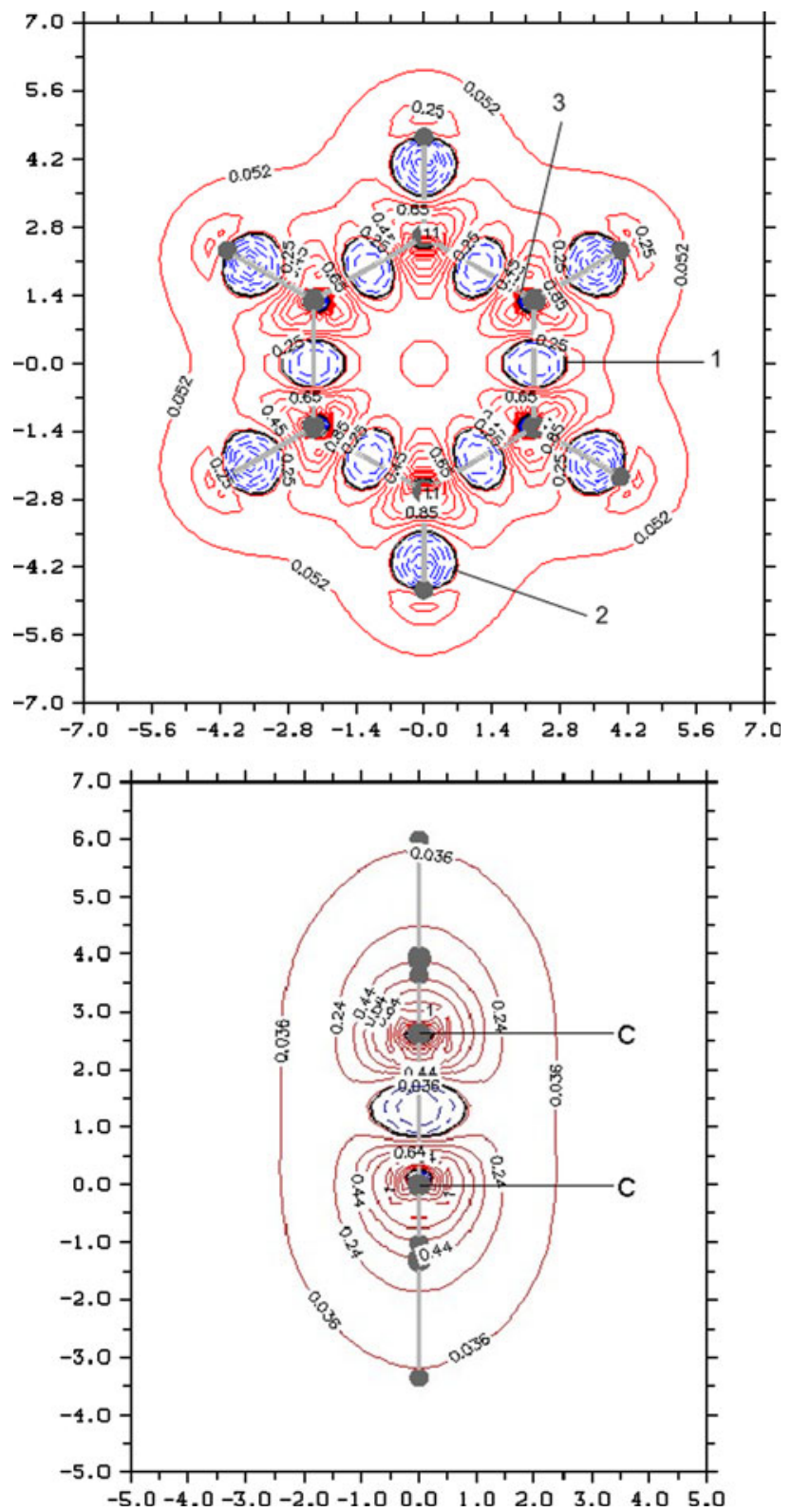

Fig. $11 \mathrm{CG}$ analysis of the chemical bonds in benzene: the molecular plane section (first panel) and perpendicular cut through the $\mathrm{C}-\mathrm{C}$ bond between the neighboring carbon atoms (second panel). The negative CG values are represented by broken lines; the same convention is used in Fig. 10 [19-21]

information density between the two nuclei. However, for more distant atomic partners, such an accumulation of valence electrons can be absent, e.g., in the cross-ring $\pi$-interactions in benzene or between the bridgehead carbon atoms in small propellanes, for which the "charge-shift" bonding mechanism [17] has been proposed within the familiar valence bond (VB) description of molecular systems. The latter involves instantaneous charge fluctuations due to a strong resonance between covalent and ionic VB structures. As we have argued elsewhere, such an indirect (implicit) bonding interaction lacking an accumulation of the bond-charge (information) can be also realized indirectly, through the neighboring $\mathrm{AO}$ intermediaries forming a bridge for an effective interaction (communication) between more distant AO.

Each pair of AO thus exhibits the partial through-space and through-bridge bond components. The "order" of the former is well reflected by the associated Wiberg contribution of Eq. 15. It quickly vanishes with increasing interatomic separation. It is also small when the interacting AO are heavily engaged in forming chemical bonds with other atoms or remain non-bonding thus describing the lone electron pairs. However, even in these directly non-bonding cases, the chemical interaction between the specified (terminal) AO can still assume appreciable values, when the remaining atoms form an effective bridge of the neighboring, chemically bonded atoms, which links the two AO in question. The Wiberg-type indices for the indirect chemical bonds have been developed [3, 48, 49] and the molecular information channels for the indirect communications in the molecular bond system, involving a single or multiple sequential "cascade" of the remaining basis functions, have been established [3,52]. These indirect channels generate the associated bridge-contributions to the overall IT-covalency (communication-noise) and IT-ionicity (information-flow) bond descriptors, which together determine the entropic measure of the overall bridge-multiplicity of the chemical bond of interest.

Thus, a non-vanishing element of the density matrix, coupling the two $\mathrm{AO}$ in the molecule, which in MO theory reflects their directly bonding status, is not essential for the existence of their through-bridge interaction. The latter may exist even when the direct interaction vanishes provided the two AO strongly couple to the chemically bonded chain of orbital intermediaries connecting them. This novel, indirect ("through-bridge") mechanism of bonding interactions in molecules, first conjectured to explain the bonding patterns of small propellane systems [1], generates the associated Wiberg-type bond-order contributions, complementary to the original Wiberg indices resulting from the familiar direct ("through-space") interactions between the basis functions used to represent the occupied molecular orbitals (MO). Together these two contributions determine the resultant multiplicities of the system chemical bonds, which in many respects provide a more balanced account of the molecular bonding patterns.

For example, this novel mechanism has been shown to have important implications for the bonding patterns of $\pi$-interactions in hydrocarbons [48, 49]. The Hückel theory description of $\pi$-bonds in benzene predicts for the neighboring ortho-carbons a strong Wiberg bond multiplicity of almost exclusively through-space origin, while the crossring interactions, between the meta- and para-carbons, where shown to be described by smaller but practically equalized resultant bond-orders, being distinguished solely 
Table 1 The resultant bond-orders (upper entry) between carbon atoms in butadiene* and benzene ${ }^{*}$ and their resolution (lower entry, in parentheses) into the direct (through space, first number) and the overall indirect (through bridges, second number) components

\begin{tabular}{|c|c|c|c|c|c|c|c|}
\hline \multirow[t]{2}{*}{ Bond } & \multirow[t]{2}{*}{ Hückel $^{\mathrm{a}}$} & \multicolumn{2}{|l|}{ RHF } & \multicolumn{2}{|c|}{ DFT: BP86 } & \multicolumn{2}{|c|}{ DFT: B3LYP } \\
\hline & & STO-nG & $631 \mathrm{G}$ & STO-nG & $631 \mathrm{G}$ & STO- $n \mathrm{G}$ & $631 \mathrm{G}$ \\
\hline \multicolumn{8}{|c|}{ Butadiene } \\
\hline \multirow[t]{2}{*}{$1-2$} & 1.83 & 1.97 & 2.08 & 1.95 & 2.05 & 1.95 & 2.06 \\
\hline & $1.80 / 0.03$ & $1.94 / 0.03$ & $2.02 / 0.06$ & $1.91 / 0.04$ & $1.99 / 0.06$ & $1.92 / 0.03$ & $2.00 / 0.06$ \\
\hline \multirow[t]{2}{*}{$1-3$} & 0.32 & 0.43 & 0.31 & 0.51 & 0.35 & 0.48 & 0.34 \\
\hline & $0.00 / 0.32$ & $0.00 / 0.43$ & $0.04 / 0.26$ & $0.00 / 0.51$ & $0.05 / 0.30$ & $0.00 / 0.48$ & $0.05 / 0.29$ \\
\hline \multirow[t]{2}{*}{$1-4$} & 0.33 & 0.23 & 0.15 & 0.31 & 0.21 & 0.29 & 0.20 \\
\hline & $0.20 / 0.13$ & $0.08 / 0.15$ & $0.09 / 0.06$ & $0.12 / 0.18$ & $0.14 / 0.08$ & $0.11 / 0.17$ & $0.12 / 0.08$ \\
\hline \multirow[t]{2}{*}{$2-3$} & 1.33 & 1.14 & 1.16 & 1.21 & 1.21 & 1.19 & 1.20 \\
\hline & $1.20 / 0.13$ & $1.06 / 0.08$ & $1.10 / 0.06$ & $1.10 / 0.11$ & $1.14 / 0.06$ & $1.09 / 0.10$ & $1.13 / 0.07$ \\
\hline \multicolumn{8}{|c|}{ Benzene } \\
\hline \multirow[t]{2}{*}{ Ortho } & 1.53 & 1.97 & 2.08 & 1.95 & 2.05 & 1.95 & 2.06 \\
\hline & $1.44 / 0.09$ & $1.94 / 0.03$ & $2.02 / 0.06$ & $1.91 / 0.04$ & $1.99 / 0.06$ & $1.92 / 0.03$ & $2.00 / 0.06$ \\
\hline \multirow[t]{2}{*}{ Meta } & 0.36 & 0.65 & 0.38 & 0.66 & 0.32 & 0.66 & 0.39 \\
\hline & $0.00 / 0.36$ & $0.00 / 0.65$ & $0.04 / 0.34$ & $0.00 / 0.66$ & $0.06 / 0.26$ & $0.00 / 0.66$ & $0.05 / 0.34$ \\
\hline \multirow[t]{2}{*}{ Para } & 0.34 & 0.48 & 0.24 & 0.49 & 0.27 & 0.49 & 0.26 \\
\hline & $0.11 / 0.23$ & $0.12 / 0.36$ & $0.12 / 0.12$ & $0.12 / 0.37$ & $0.13 / 0.14$ & $0.12 / 0.37$ & $0.13 / 0.13$ \\
\hline
\end{tabular}

* For the common equilibrium geometry in $631 \mathrm{G}$ basis set

* For the common equilibrium geometry in TZV basis set

a To facilitate a comparison, the single bond multiplicity for the missing $\sigma$ bond was added

by their direct/indirect composition. The meta bonds have been shown to be realized exclusively through bridges, while the para bonds exhibit a comparable direct and indirect components.

In this section, we examine the numerical predictions of the Wiberg-type bond-orders for such indirect chemical bonds resulting from the all-electron SCF MO (RHF) and DFT (BP86 and B3LYP) calculations for benzene and butadiene in the two representative Gaussian basis sets: the minimum (STO- $n \mathrm{G}$ ) and extended (631G) bases of molecular calculations (Table 1). These predictions will be compared with the previous findings for the Hückel approximation. In order to test the range of such implicit chemical interactions in larger systems, the interaction of the terminal and interior atoms separated by increasing segments of the representative polymer chains consisting of 15 heavy atoms: in polyen, polyethylene, polybatadiene, each including 15 carbon atoms, and in polyglycine (10 carbons and 5 nitrogens) have been investigated (Table 2).

The Wiberg index of the direct bond-order between the specified pair $(\mathrm{X}, \mathrm{Y})$ of AIM is given by the sum of the two AO contributions of Eq. 15:

$M_{\mathrm{X}, \mathrm{Y}}=\sum_{x \in \mathrm{X}} \sum_{y \in \mathrm{Y}} M_{x, y}$.

It has been shown elsewhere [48, 49] that the natural extension of this two AO bond multiplicity into the
Wiberg-type index for the indirect bond realized via the single- or multiple-AO bridges is given by the product of the elementary bond-orders of the direct chemical interactions along the bridge. For example, for the singleAO bridges realized by orbitals $|k\rangle=\left\{\chi_{k} \notin(\mathrm{X}, \mathrm{Y})\right\}$, i.e., by the single-AIM bridges of the remaining atoms $\{\mathrm{Z} \neq(\mathrm{X}, \mathrm{Y})\}$, the indirect bond-order contributions

$M_{\mathrm{X}, \mathrm{Y}}(\boldsymbol{k})=\sum_{x \in \mathrm{X}} \sum_{y \in \mathrm{Y}} \sum_{k \notin(\mathrm{X}, \mathrm{Y})} M_{x, k} M_{k, y} \equiv \sum_{k \notin(\mathrm{X}, \mathrm{Y})} M_{\mathrm{X}, k} M_{k, \mathrm{Y}}$,

while the double-AO bridges realized by orbitals $\left.(|\boldsymbol{k}\rangle,|\boldsymbol{l}\rangle)=\left\{\left(\chi_{k}, \chi_{l}\right)\right\} \notin(\mathrm{X}, \mathrm{Y})\right\}$, i.e., by the double-AIMbridges $\{R, Z\} \neq\{X, Y\}$, give the associated indirect bond-order

$$
\begin{aligned}
M_{\mathrm{X}, \mathrm{Y}}(\boldsymbol{k}, \boldsymbol{l}) & =\sum_{x \in \mathrm{X}} \sum_{y \in \mathrm{Y}} \sum_{k \notin(\mathrm{X}, \mathrm{Y})} \sum_{l \notin(\mathrm{X}, \mathrm{Y})} M_{x, k} M_{k, l} M_{l, y} \\
& =\sum_{k \notin(\mathrm{X}, \mathrm{Y})} \sum_{l \notin(\mathrm{X}, \mathrm{Y})} M_{\mathrm{X}, k} M_{k, l} M_{l, \mathrm{Y}} .
\end{aligned}
$$

These expressions can be straightforwardly extended to cover general cases of more orbitals in the bridge connecting the specified pair of terminal AO. The indirect bond-orders and the associated resultant multiplicities reported in Table 1 reflect the most important single- and double-AO bridges in the two hydrocarbons, while the multiple-AO bridges in Table 2 are realized only via a chain of bonded 
Table 2 The direct (Wiberg) and indirect (through the polymer segment) bond-orders in illustrative polymers (from RHF calculations using the minimum basis set)

\begin{tabular}{|c|c|c|c|c|c|c|c|c|}
\hline \multirow[t]{2}{*}{ Bond $^{\mathrm{a}}$} & \multicolumn{2}{|c|}{ Polyene } & \multicolumn{2}{|c|}{ Polyethylene } & \multicolumn{2}{|c|}{ Polybutadiene } & \multicolumn{2}{|c|}{ Polyglycine } \\
\hline & Direct & Bridge & Direct & Bridge & Direct & Bridge & Direct & Bridge \\
\hline $1-2$ & 1.930 & & 1.014 & & 1.017 & & 1.023 & \\
\hline $1-3$ & 0.003 & 0.465 & 0.002 & 0.370 & 0.004 & 0.411 & 0.005 & 0.339 \\
\hline $1-4$ & 0.076 & 0.214 & 0.007 & 0.134 & 0.012 & 0.141 & 0.005 & 0.111 \\
\hline $1-5$ & 0.000 & 0.061 & 0.001 & 0.049 & 0.001 & 0.050 & 0.001 & 0.041 \\
\hline $1-6$ & 0.013 & 0.026 & 0.000 & 0.018 & 0.000 & 0.017 & 0.000 & 0.014 \\
\hline $1-7$ & 0.000 & 0.008 & 0.000 & 0.006 & 0.000 & 0.007 & 0.000 & 0.004 \\
\hline $1-8$ & 0.003 & 0.003 & 0.000 & 0.002 & 0.000 & 0.002 & 0.000 & 0.004 \\
\hline $1-9$ & 0.000 & 0.001 & 0.000 & 0.001 & 0.000 & 0.001 & 0.000 & 0.002 \\
\hline
\end{tabular}

${ }^{a}$ Between indicated atoms in the polymer chain, for their consecutive numbering. In polyglycine, the nitrogen atom represents the first atom of the chain

atoms in the polymer segment which separates the current choice of terminal AO/AIM.

It follows from Table 1 that in general the resultant bond multiplicities from all-electron calculations compare favorably with the model predictions from Hückel's theory. Only a weak basis set dependence is detected, with extended basis generating slightly higher resultant multiplicities. The resultant bond multiplicities reflect a more natural gradation of relative bond strengths with increasing distance between the directly non-bonded carbon atoms, with the ortho-carbons, the nearest-neighbors in the benzene ring, forming the strongest chemical bonds, and the most distant (thirdneighbor) para-carbons now being predicted to exibibit the weakest cross-ring bonds, smaller than that for the (secondneighbor) meta-carbons, which remain directly $\pi$ nonbonding. A similar trend trinspitres from the overall bond multiplicities for carbon-carbon chemical bonds in butadiene: in both basis sets the 1-3 interaction is predicted to be stronger than the resultant 1-4 bond. The resultant indices for the strongest bonds in each system, now giving approximately double bond multiplicity, slightly higher that the fractional data of Hückel approximation, which more closely reflect the intuitive chemical values.

The bond patterns exhibited in Table 2 by the direct and indirect measures of the effective interactions between AIM separated by the bridge of intervening fragment of the polymer chain explicitly demonstrate the short-range of the former and distinctly longer range of the latter. Indeed the Wiberg bond-orders fast decay with increasing bridge length, while their bridge analogs exhibit an appreciable component up to bridge length of five atoms. The selection of illustrative polymers generates a variety of $\pi$ bonds and their mutual coupling in the system and the associated bridges. Again, in the direct bond-orders a non-monotonic behavior is observed, while the bridge terms constitute a smooth sequence of monotonically decaying bond-orders. For a single atom in the bridge, the polyene is seen to generate the highest bridge bond-order, due to a strongly conjugated nature of the alternating double bonds. It follows from the Hückel theory that this alternation is strongest at both ends of the polyene, with almost equalized bond-orders in the polyene interior. A similar conclusion follows from an earlier IT analysis of a very long polyene in the Hückel approximation [52].

\section{Ionic promotion of reactants by a catalyst}

The molecular scenario invoking IT quantities of three probability distributions (see the second part of Fig. 1) may involve three separate species $A, B$, and $C$, e.g., two reactants $\mathrm{A}$ and $\mathrm{B}$ and the catalyst/surface $\mathrm{C}$, with the corresponding sets of the AO-events $(\boldsymbol{a}, \boldsymbol{b}, \boldsymbol{c})$ of the associated probability schemes $(\mathrm{A}, \mathrm{B}, \mathrm{C})$ then referring to the basis functions provided by the constituent atoms in each of these subsystems. Alternatively, three molecular fragments can be involved. The three-orbital development in OCT then enables one to discuss the influence of one reactant/fragment, say $\mathrm{C}$, on the bond structure (or reactivity) of two remaining fragments $\mathrm{A}$ and $\mathrm{B}[2,3,64]$. For example, one could then address a natural question: how the electronic/bonding structure of the catalyst affects the structure/reactivity patterns of two adsorbed species, and ultimately assess the cooperation effects between the catalyst-adsorbate bonds $(\mathrm{A}-\mathrm{C}, \mathrm{B}-\mathrm{C})$ and the A-B bond linking the two adsorbates. Alternatively, one could tackle in this way the influence of the chemical reactivity on one active site on that exhibited by the other site of the surface.

In this three-scheme framework, the product probability schemes $\alpha=\mathrm{AC}$ and $\beta=\mathrm{BC}$ of the chemisorbed reactants, corresponding to the joint probabilities 
$\pi(\boldsymbol{a} \wedge \boldsymbol{c}) \equiv \mathbf{P}(\boldsymbol{\alpha})$ and $\pi(\boldsymbol{b} \wedge \boldsymbol{c}) \equiv \mathbf{P}(\boldsymbol{\beta})$, respectively, effectively extend the entropy areas of the adsorbed species (see Fig. 1):

$S(\boldsymbol{\pi}(\boldsymbol{a} \wedge \boldsymbol{c})) \equiv S(\mathrm{AC})=S(\alpha)>S(\mathrm{~A}) \quad$ and $S(\boldsymbol{\pi}(\boldsymbol{b} \wedge \boldsymbol{c})) \equiv S(\mathrm{BC})=S(\beta)>S(\mathrm{~B})$.

Hence, the effective IT-covalency of interactions between the chemisorbed reactants decreases compared to their coupling in the gas phase:

$$
\begin{aligned}
S(\beta \mid \alpha) & =S(\boldsymbol{P}(\boldsymbol{b}) \mid \boldsymbol{\pi}(\boldsymbol{a} \wedge \boldsymbol{c})) \equiv S(\mathrm{~B} \mid \mathrm{AC}) \\
& =S(\mathrm{~B} \mid \mathrm{A})-I(\mathrm{~B}: \mathrm{C} \mid \mathrm{A})<S(\mathrm{~B} \mid \mathrm{A}),
\end{aligned}
$$

while the effective IT-ionicity of the chemisorbed species dramatically increases due to the catalyst:

$I(\alpha: \beta) \equiv S(\mathrm{C})+I(\mathrm{~A}: \mathrm{B} \mid \mathrm{C})>I(\mathrm{~A}: \mathrm{B})$.

This net IT-ionic "activation" of adsorbates, as a result of forming partial A-C and B-C bonds on active sites of the catalytic surface, also manifests the competition effect between these surface bonds and the inter-adsorbate bond A-B in the catalytic system: the more heavily are the valence electrons of $\mathrm{A}$ and $\mathrm{B}$ involved in chemical bonds with $\mathrm{C}$, the less noisy (more deterministic) are their mutual communications, thus giving rise to less IT-covalent (more IT-ionic) interactions between the chemisorbed species. The physical adsorption of these reactants should be marked by a relatively small value of $I(\mathrm{~B}: \mathrm{ClA})$, since then the dependencies ("overlaps") between entropy circles of schemes (B, A) and C, respectively, should be small thus grossly diminishing this IT-ionic activation effect generated by the catalyst presence. Indeed, the surface chemical bond between a given adsorbate and the catalyst should be strongly felt at the position of the other adsorbate, and hence the information "coupling" between probability distributions of the chemisorbed species and that of the catalyst should be relatively strong.

Another molecular scenario, in which the three-scheme entropy/information descriptors are expected to be useful, is the influence of one reactive site in a molecule upon another, e.g., in the contexts of subtle reactivity preferences in the donor-acceptor (DA) reactive systems implied by the hard and soft acids and bases (HSAB) rule, and particularly-its regional formulation for predicting the regioselectivity trends in cyclization reactions, the maximum complementarity principle, and the bond-length variation rules of Gutmann. Such cooperative interaction between different sites in a single molecule is also responsible for the directing trans/cis influence of ligands in transition metal complexes and the familiar substituent effect in aromatic systems. The adequate IT description of the AIM cooperation in many-centre bonds, e.g., in boron hydrides or propellanes, may also require the entropy/information indices involving several probability schemes.

\section{Conclusion}

In this study, we have emphasized the additional possibilities in the electronic structure theory offered by IT, and particularly by OCT, in exploring the molecular patterns of chemical bonds and understanding the mutual competition of its diverse atomic and diatomic components. This new perspective, complementary to the familiar MO description, was shown to bring important new insights. It enhances our understanding of the always fascinating phenomenon of the chemical bond and reactivity. On one hand, it generates new probes for detecting the direct bonding interactions in the system electron distribution. On the other hand, the IT approach uncovers the bond entropic origins and explains the direct and indirect sources of its resultant multiplicity.

The use of maps of the entropy-displacement and entropy-deficiency densities as well as of the non-additive Fisher information, the basis of the CG criterion of bond localization, in probing the chemical bonds was emphasized and illustrated for selected molecules. The complementary entropy/information bond components, measuring the communication-noise (IT-covalency) and informationflow (IT-ionicity) between orbitals in molecular information channels have been related to similar concepts in $\mathrm{MO}$ theory. The IT analysis was also shown to provide a resolution of the (localized) bond-order concept of Wiberg in MO theory into such entropic components. This communication theory clearly distinguishes between the direct ("conversation") and indirect ("gossip") sources of chemical interactions in IT, which in MO approach result from the implicit dependencies between the (non-orthogonal) AO projections onto the bond-subspace of the molecular Hilbert space of SCF LCAO MO calculations.

In this overview, we have also stressed the basic equivalence of the entropic and MO treatments of the chemical "bond-orders." The Wiberg bond multiplicity, which unifies these two perspectives, was extended to cover the indirect bond components, realized through the orbital intermediaries. This new mechanism was shown to extend the effective range of chemical interactions. The overall bond indices were shown to provide a more balanced account of the cross-ring $\pi$ bonds in benzene, and of the second-neighbor interactions in butadiene. They also explain the origins of the chemical interactions in small propellanes. We have also tested the effective range of the indirect chemical interactions in illustrative polymers.

The entropy/information descriptors of the coupling between fragments of molecules or reactive systems involve descriptors of several probability distributions. Such concepts and the underlying communication systems have recently been introduced in OCT. They open new possibilities in describing diverse phenomena of conjugations 
between chemical bonds and/or reactivity sites. In this study, we have briefly demonstrated how such generalized entropic quantities of IT can be employed to examine the reactivity of the chemisorbed reactants in catalysis. This qualitative analysis indicates that the communications between such "promoted" states of the adsorbates become distinctly more deterministic (IT-ionic) compared to those between reactants in the gas phase.

One should finally mention the unifying character of the IT variational rules in physics [1-3, 14, 15], and particularly in the molecular quantum mechanics. They are vital for an unbiased extraction of molecular fragments, e.g., the bonded atoms [1]. These principles introduce the thermodynamic-like, phenomenological description of molecular equilibria and enhance our understanding of the behavior of open molecular subsystems in reactivity phenomena [1-3].

Open Access This article is distributed under the terms of the Creative Commons Attribution License which permits any use, distribution, and reproduction in any medium, provided the original author(s) and the source are credited.

\section{References}

1. Nalewajski RF (2006) Information theory of molecular systems. Elsevier, Amsterdam

2. Nalewajski RF (2010) Information origins of the chemical bond. Nova Science Publishers, New York

3. Nalewajski RF (2012) Perspectives in electronic structure theory. Springer, Heidelberg

4. Nalewajski RF, Broniatowska E (2003) J Phys Chem A 107:6270

5. Nalewajski RF, Świtka E, Michalak A (2002) Int J Quantum Chem 87:198

6. Nalewajski RF, Świtka E (2002) Phys Chem Chem Phys 4:4952

7. Shannon CE (1948) Bell System Tech J 27(379):623

8. Shannon CE, Weaver W (1949) The mathematical theory of communication. University of Illinois, Urbana

9. Abramson N (1963) Information theory and coding. McGrawHill, New York

10. Pfeifer PE (1978) Concepts of probability theory, 2nd edn. Dover, New York

11. Kullback S, Leibler RA (1951) Ann Math Stat 22:79

12. Kullback S (1959) Information theory and statistics. Wiley, New York

13. Fisher RA (1925) Proc Cambridge Phil Soc 22:700

14. Frieden BR (2004) Physics from the Fisher information-a unification. Cambridge University Press, Cambridge

15. Nalewajski RF (2008) Int J Quantum Chem 108:2230

16. Hirshfeld FL (1977) Theoret Chim Acta (Berlin) 44:129

17. Shaik S, Danovich D, Wu W, Hiberty PC (2009) Nat Chem 1:443

18. Nalewajski RF (2011) J Math Chem 49:546

19. Nalewajski RF, de Silva P, Mrozek J (2012) In: Roy AK (ed) Theoretical and computational developments in modern density functional theory, Nova Science Publishers, New York (in press)
20. Nalewajski RF (2010) J Math Chem 47:667

21. Nalewajski RF, de Silva P, Mrozek J (2010) J Mol Struct (Theochem) 954:57

22. Nalewajski RF, Köster AM, Escalante S (2005) J Phys Chem A 109:10038

23. Becke AD, Edgecombe KE (1990) J Chem Phys 92:5397

24. Silvi B, Savin A (1994) Nature 371:683

25. Savin A, Nesper R, Wengert S, Fässler TF (1997) Angew Chem Int Ed Engl 36:1808

26. Nalewajski RF (2009) Int J Quantum Chem 109:425

27. Nalewajski RF (2009) Int J Quantum Chem 109:2495

28. Nalewajski RF (2009) Adv Quantum Chem 56:217

29. Nalewajski RF (2010) J Math Chem 47:709

30. Nalewajski RF (2010) J Math Chem 47:692

31. Nalewajski RF (2011) J Math Chem 49:592

32. Nalewajski RF (2011) J Math Chem 49:2308

33. Nalewajski RF, Szczepanik D, Mrozek J (2011) Adv Quantum Chem 61:1-48

34. Nalewajski RF, Szczepanik D, Mrozek J (2012) J Math Chem 50:1437

35. Nalewajski RF (2000) J Phys Chem A 104:11940

36. Dirac PAM (1958) The principles of quantum mechanics, 4th edn. Clarendon, Oxford

37. Wiberg KA (1968) Tetrahedron 24:1083

38. Gopinathan MS, Jug K (1983) Theor Chim Acta (Berl) 63(497):511

39. Jug K, Gopinathan MS (1990) In: Maksić ZB (ed) Theoretical models of chemical bonding, vol II. Springer, Heidelberg, p 77

40. Mayer I (1983) Chem Phys Lett 97:270

41. Nalewajski RF, Köster AM, Jug K (1993) Theor Chim Acta (Berl) 85:463

42. Nalewajski RF, Mrozek J (1994) Int J Quantum Chem 51:187

43. Nalewajski RF, Formosinho SJ, Varandas AJC, Mrozek J (1994) Int J Quantum Chem 52:1153

44. Nalewajski RF, Mrozek J, Mazur G (1996) Can J Chem 100:1121

45. Nalewajski RF, Mrozek J, Michalak A (1997) Int J Quantum Chem 61:589

46. Mrozek J, Nalewajski RF, Michalak A (1998) Polish J Chem 72:1779

47. Nalewajski RF (2004) Chem Phys Lett 386:265

48. Nalewajski RF (2011) J Math Chem 49:371

49. Nalewajski RF (2011) J Math Chem 49:546

50. Nalewajski RF (2011) J Math Chem 49:806

51. Nalewajski RF, Gurdek P (2011) J Math Chem 49:1226

52. Nalewajski RF (2012) Int J Quantum Chem 112:2355

53. von Weizsäcker CF (1935) Z Phys 96:431

54. Nalewajski RF (2005) Mol Phys 103:451

55. Nalewajski RF (2006) Mol Phys 104:493

56. Gordon RG, Kim YS (1972) J Chem Phys 56:3122

57. Cortona P (1991) Phys Rev B 44:8454

58. Wesołowski TA, Warshel A (1993) J Phys Chem 97:8050

59. Wesołowski TA, Muller RP, Warshel A (1995) J Phys Chem 100:15444

60. Wesołowski TA, Weber J (1998) Chem Phys Lett 248:71

61. Wesołowski TA, Tran F (2003) J Chem Phys 118:2072

62. Wesołowski TA (2004) J Am Chem Soc 126:11444

63. Wesołowski TA (2004) Chimia 58:311

64. Nalewajski RF (2010) J Math Chem 47:808 